\title{
Discrete Fractional COSHAD Transform and Its Application
}

\author{
Hongqing Zhu, ${ }^{1}$ Zhiguo Gui, ${ }^{2}$ Yu Zhu, ${ }^{1}$ and Zhihua Chen ${ }^{1}$ \\ ${ }^{1}$ School of Information Science \& Engineering, East China University of Science and Technology, Shanghai 200237, China \\ ${ }^{2}$ National Key Laboratory for Electronic Measurement Technology, North University of China, Taiyuan 030051, China
}

Correspondence should be addressed to Hongqing Zhu; hqzhu@ecust.edu.cn

Received 25 March 2014; Revised 2 June 2014; Accepted 18 June 2014; Published 24 July 2014

Academic Editor: Kui Fu Chen

Copyright (C) 2014 Hongqing Zhu et al. This is an open access article distributed under the Creative Commons Attribution License, which permits unrestricted use, distribution, and reproduction in any medium, provided the original work is properly cited.

\begin{abstract}
In recent years, there has been a renewed interest in finding methods to construct orthogonal transforms. This interest is driven by the large number of applications of the orthogonal transforms in image analysis and compression, especially for colour images. Inspired by this motivation, this paper first introduces a new orthogonal transform known as a discrete fractional COSHAD (FrCOSHAD) using the Kronecker product of eigenvectors and the eigenvalues of the COSHAD kernel functions. Next, this study discusses the properties of the FrCOSHAD kernel function, such as angle additivity. Using the algebra of quaternions, the study presents quaternion COSHAD/FrCOSHAD transforms to represent colour images in a holistic manner. This paper also develops an inverse polynomial reconstruction method (IPRM) in the discrete COSHAD/FrCOSHAD domains. This method can effectively recover a piecewise smooth signal from the finite set of its COSHAD/FrCOSHAD coefficients, with high accuracy. The convergence theorem has proved that the partial sum of COSHAD provides a spectrally accurate approximation to the underlying piecewise smooth signal. The experimental results verify the numerical stability and accuracy of the proposed methods.
\end{abstract}

\section{Introduction}

With the development of fast algorithms, the discrete cosine transform (DCT) and the Hadamard transform have been widely used in signal processing and image processing, most prominently in the compressed representations of images [13]. The DCT is a real-to-real and integer-to-real transform that has been considered as an alternative to the Fourier transform for spectral analysis. The DCT has the additional advantage that its forward and inverse forms are identical, thus, simplifying implementation. The Hadamard transform is highly practical for representing signals, images, and mobile communications, mainly because the elements of the Hadamard matrix are either +1 or -1 . Thus, the computation of the transform of a signal consists of additions and subtractions of the signal samples. The Hadamard transform and many of its variations, such as the sequency-ordered complex Hadamard transform (SCHT) $[4,5]$ and the Jacket transform [6], have been proposed, and their applications to image processing and communications have been reported [7].

In recent decades, various factional series and transforms have been introduced and have found application in many fields in engineering [8-11]. In [10], Pei and Yeh developed a recursive method to obtain Hadamard eigenvectors, and normalised eigenvectors were used to define the discrete fractional Hadamard transform. As an alternative to the recursive method, Tseng [12] investigated the eigenstructure of the Hadamard transform for defining its fractional transform and found that the Kronecker product is more suitable for calculating the eigenvalues and the eigenvectors of the Hadamard kernel function.

As another application of the Kronecker product, the COSHAD kernel function was first introduced by Merchant and Rao [13]. COSHAD stands for a hybrid version of discrete Cosine and Hadamard transform. The COSHAD kernel function is also a hybrid function derived from the Kronecker product of the DCT and Hadamard kernel functions. The motivation for developing the COSHAD transform is to compromise between these two transforms in terms of implementation, properties, and applications. However, the study of COSHAD is far from complete and mainly limited within its fast algorithms and filtering applications in the same paper [13]. Although these transforms are remarkable as a type of discrete orthogonal transform, the signal and 
image representation abilities of COSHAD have not been investigated. Almost no literature addresses the energy compaction of COSHAD. Furthermore, the motivation behind the development of the COSHAD is to seek the combination of the advantages of the two transforms. How can the investigator compromise between these two transforms according to performance and computational burden? What type of impact parameter $m$ is produced in the COSHAD kernel function? Further study of the COSHAD transform is, therefore, necessary.

The objective of this paper is twofold. First, we report a relatively complete study of the representation capabilities of the COSHAD transform. Another new orthogonal transform, named the fractional COSHAD (FrCOSHAD) transform, and its properties are proposed from the eigendecomposition of the COSHAD kernel function. The FrCOSHAD is a generalisation of the COSHAD transform that will reduce to the COSHAD transform when the fractional order $p$ of the FrCOSHAD kernel function is unity. The advantage of the generalisation is that the FrCOSHAD contains more parameters and is, therefore, more flexible for whatever application it will be used for. Besides, this paper defines the so-called quaternion FrCOSHAD (Q-FrCOSHAD) to encode colour images as vector fields and investigates their energy compaction properties theoretically. The advantage of such a representation is that a colour image can be treated in a holistic manner, and each pixel can be handled as a vector.

Second, we represent a signal from a limited number of terms in the partial sum of the COSHAD/FrCOSHAD series with high accuracy. The number of terms in the expansion is known to be increased; the series approximation converges in the region where the function is smooth. However, the overshoot near the discontinuity is never reduced, no matter how many terms are included in the partial sum. Furthermore, the numerical requirements grow dramatically with the increase in the number of harmonics. The expectation, in practice, is that the signal could be reconstructed from much less data at a high level of quality. To solve this problem, this study follows the main description of the inverse polynomial reconstruction method (IPRM) for the continuous Fourier series expansion [14-16] and derives a mathematics framework of the IPRM for the discrete COSHAD/FrCOSHAD series expansion to accurately recover a piecewise smooth signal. The spectral convergence of the proposed IPRM strategy in the discrete COSHAD expansion is discussed and proven, both theoretically and numerically.

The remainder of this paper is organised as follows. Section 2 provides a brief review of the discrete COSHAD transform. Section 3 introduces the discrete FrCOSHAD transform and its main properties. The quaternion discrete COSHAD/FrCOSHAD transforms based on quaternion algebra are also defined in this section. The derivation of the COSHAD-based ID IPRM and the convergence properties are presented in Section 4. Section 5 reports the simulation results for the standard testing images and provides the numerical verification of the convergence theorems. Finally, in Section 6, we summarise the results and conclude the paper.

\section{Review of the COSHAD Transform}

This section is devoted to a review of the results about the discrete COSHAD transform defined in [13], which may not be widely known. We start by defining the 1D forward COSHAD transform of order $m$ in the following form:

$$
\mathbf{Y}=\frac{1}{\sqrt{N}}\left[\operatorname{CosHAD}_{m}(n)\right] \mathbf{X},
$$

where $n=\log _{2}(N), N$ is the length of signal $\mathbf{X}, \mathbf{X}^{T}=$ $[x(0), x(1), \ldots, x(N-1)]$ is the data vector, and $\mathbf{Y}^{T}=$ $[y(0), y(1), \ldots, y(N-1)]$ is the transform vector. The $m$ th order $2^{n} \times 2^{n}$ COSHAD kernel function (transform matrix) is defined as

$$
\left[\operatorname{COSHAD}_{m}(n)\right]=[\mathrm{DCT}(m)] \otimes[\operatorname{HAD}(n-m)],
$$

and the symbol $\otimes$ represents the Kronecker product. $[\operatorname{DCT}(m)]$ and $[\operatorname{HAD}(n-m)]$ are DCT (of size $2^{m} \times 2^{m}$ ) and Hadamard kernel functions (of size $2^{n-m} \times 2^{n-m}$ ), respectively. In this paper, we rely heavily on Corollary 13.8 in [17] as follows.

Corollary 1. If $\mathbf{A} \in \mathbf{R}^{n \times n}$ is orthogonal and $\mathbf{B} \in \mathbf{R}^{m \times m}$ is orthogonal, then $\mathbf{A} \otimes \mathbf{B}$ is orthogonal.

According to Corollary 1 , the defined $\left[\operatorname{COSHAD}_{m}(n)\right]$ is an orthogonal kernel function that belongs to a family of discrete orthogonal matrices ranging from the Hadamard kernel function $(m=0)$ to the DCT kernel function $(m=n)$. Thus, the inverse COSHAD transform $\mathbf{X}$ is defined as

$$
\mathbf{X}=\frac{1}{\sqrt{N}}\left[\operatorname{COsHAD}_{m}(n)\right]^{T} \mathbf{Y},
$$

where $T$ denotes the matrix transpose. The $2 \mathrm{D}$ forward COSHAD transform of an $N \times N$ matrix $\mathbf{f}$ is defined by

$$
\mathbf{F}=\left[\frac{1}{\sqrt{N}} \operatorname{COsHAD}_{m}(n)\right] \mathbf{f}\left[\frac{1}{\sqrt{N}} \operatorname{COSHAD}_{m}(n)\right]^{T} .
$$

The corresponding inverse COSHAD transform can be calculated as

$$
\mathbf{f}=\left[\frac{1}{\sqrt{N}} \operatorname{CosHAD}_{m}(n)\right]^{T} \mathbf{F}\left[\frac{1}{\sqrt{N}} \operatorname{CosHAD}_{m}(n)\right] .
$$

\section{The Discrete FrCOSHAD Transform and Its Properties}

3.1. The Discrete FrCOSHAD Transform. According to the Kronecker product properties [17] and the definition of the Hadamard transform, one can rewrite the Hadamard kernel function as follows:

$$
[\operatorname{HAD}(n)]=[\operatorname{HAD}(1)]^{\otimes n} .
$$

If the lowest-order Hadamard kernel function $[\mathrm{HAD}(1)]$ is expressed as

$$
[\operatorname{HAD}(1)]=\left[\begin{array}{cc}
1 & 1 \\
1 & -1
\end{array}\right]
$$


Substituting (6) into (2), the COSHAD kernel function can be rewritten as

$$
\left[\operatorname{CosHAD}_{m}(n)\right]=[\operatorname{DCT}(m)] \otimes[\operatorname{HAD}(1)]^{\otimes(n-m)} .
$$

If the eigendecompositions of the matrices $[\mathrm{DCT}(m)]$ and $[\operatorname{HAD}(1)]$ are

$$
\begin{aligned}
& {[\operatorname{DCT}(m)]=V_{1} D_{1} V_{1}^{-1},} \\
& {[\operatorname{HAD}(1)]=V_{2} D_{2} V_{2}^{-1},}
\end{aligned}
$$

respectively, $V_{1}$ and $V_{2}$ are the matrices composed of eigenvectors. We also need to use the following properties of the Kronecker, which are 13.12 in [17].

Property 1. Let $\mathbf{A} \in \mathbf{R}^{n \times n}$ have eigenvalues $\lambda_{i}, i=1, \ldots, n$, and let $\mathbf{B} \in \mathbf{R}^{m \times m}$ have eigenvalues $\mu_{j}, j=1, \ldots, m$. Then, the $m n$ eigenvalues of $\mathbf{A} \otimes \mathbf{B}$ are $\lambda_{1} \mu_{1}, \ldots, \lambda_{1} \mu_{m}$, $\lambda_{2} \mu_{1}, \ldots, \lambda_{2} \mu_{m}, \ldots, \lambda_{n} \mu_{m}$.

Therefore, the eigendecomposition of $\left[\operatorname{COSHAD}_{m}(n)\right]$ can be written in the following form:

$$
\begin{aligned}
& {\left[\operatorname{COSHAD}_{m}(n)\right]} \\
& \quad=\left(V_{1} D_{1} V_{1}^{-1}\right) \otimes\left(V_{2} D_{2} V_{2}^{-1}\right)^{\otimes(n-m)} \\
& =\left(V_{1} \otimes V_{2}^{\otimes(n-m)}\right)\left(D_{1} \otimes D_{2}^{\otimes(n-m)}\right)\left(V_{1} \otimes V_{2}^{\otimes(n-m)}\right)^{-1} \\
& \quad=V_{N} D_{N} V_{N}^{-1},
\end{aligned}
$$

where $n=\log _{2}(N), V_{N}=V_{1} \otimes V_{2}^{\otimes(n-m)}$ and $D_{N}=D_{1} \otimes$ $D_{2}^{\otimes(n-m)}$. After the eigenvalues of the $\left[\operatorname{COSHAD}_{m}(n)\right]$ are determined, we define the kernel function of the discrete FrCOSHAD transform by taking the fractional powers $p$ of the eigenvalue.

Consider

$$
\mathrm{CH}_{N}^{p}=\left[\operatorname{COSHAD}_{m}(n)\right]^{p}=V_{N} D_{N}^{p} V_{N}^{-1} .
$$

Thus, the $1 \mathrm{D}$ forward FrCOSHAD transform with parameter $p$ is

$$
\mathbf{Y}=\left(\frac{1}{\sqrt{N}}\right)^{p} \mathrm{CH}_{N}^{p} \mathbf{X}=\left(\frac{1}{\sqrt{N}}\right)^{p} V_{N} D_{N}^{p} V_{N}^{-1} \mathbf{X} .
$$

Equation (12) indicates that the FrCOSHAD transform reduces to the standard COSHAD transform if the fractional powers $p=1$. The corresponding inverse FrCOSHAD transform is written as

$$
\mathbf{X}=\left(\frac{1}{\sqrt{N}}\right)^{-p} \mathrm{CH}_{N}^{-p} \mathbf{Y} .
$$

Similarly, the 2D forward FrCOSHAD transform with order $(p, q)$ is given by

$$
\mathbf{F}=\left[\frac{1}{\sqrt{N}} \operatorname{CosHAD}_{m}(n)\right]^{p} \mathbf{f}\left(\left[\frac{1}{\sqrt{N}} \operatorname{CoshAD}_{m}(n)\right]^{q}\right)^{T} .
$$

The corresponding inverse FrCOSHAD transform can be generated as

$$
\mathbf{f}=\left[\frac{1}{\sqrt{N}} \operatorname{CosHAD}_{m}(n)\right]^{-p} \mathbf{F}\left(\left[\frac{1}{\sqrt{N}} \operatorname{CoshAD}_{m}(n)\right]^{-q}\right)^{T} .
$$

3.2. Properties of the Discrete FrCOSHAD Kernel Function. This subsection presents some properties of the discrete FrCOSHAD kernel function.

Property 2. Additivity:

$$
\mathrm{CH}_{N}^{p_{1}} \mathrm{CH}_{N}^{p_{2}}=\mathrm{CH}_{N}^{p_{1}+p_{2}} \text {. }
$$

Proof. From (11), we have

$$
\begin{aligned}
& \mathrm{CH}_{N}^{p_{1}} \mathrm{CH}_{N}^{p_{2}} \\
& \quad=V_{N} D_{N}^{p_{1}} V_{N}^{-1} V_{N} D_{N}^{p_{2}} V_{N}^{-1}=V_{N} D_{N}^{p_{1}} D_{N}^{p_{2}} V_{N}^{-1} \\
& \quad=V_{N} D_{N}^{p_{1}+p_{2}} V_{N}^{-1}=\mathrm{CH}_{N}^{p_{1}+p_{2}} .
\end{aligned}
$$

The proof is complete.

Because of the additivity property of the FrCOSHAD kernel function, an FrCOSHAD transform with a fractional order $p_{1}$ operated on by an FrCOSHAD transform with a fractional order $p_{2}$ will be an FrCOSHAD transform with fractional order $\left(p_{1}+p_{2}\right)$. To evaluate this one basic feature of the FrCOSHAD kernel function, we investigate the performance of the $1 \mathrm{D}$ FrCOSHAD transform using a discrete rectangular window function defined as

$$
x(t)= \begin{cases}1, & \text { for }|t| \leq 50, \\ 0, & \text { for } 51 \leq|t| \leq 120,\end{cases}
$$

a triangular pulse function of width 100 and the 180th row of the standard gray-level image "Lena" of size $256 \times 256$ from the USC-SIPI image database [18] shown in Figure 1(a). These functions are calculated using the 1D FrCOSHAD transform, and the magnitudes of the resulting outputs with different values of $p$ are shown in Table 1 . The values of fractional order $p$ are taken from the set $\{0.1,0.3,0.4,0.7\}$. The last row of Table 1 is an FrCOSHAD transform $\mathrm{CH}_{N}^{0.1}$ with $p=0.1$ performed by an FrCOSHAD transform $\mathrm{CH}_{N}^{0.3}$ with $p=0.3$. The transformed results are very close to the FrCOSHAD transformed results with $p=0.4$. This result indicates that the angle additivity property holds for the $1 \mathrm{D}$ FrCOSHAD transform. Moreover, as Table 1 shows, with the increase of the fractional order $p$, the influence produced by FrCOSHAD becomes more and more obvious.

Property 3. Unitarity:

$$
\mathrm{CH}_{N}^{-p}=\left(\mathrm{CH}_{N}^{p}\right)^{-1} .
$$

Proof. Using (11), we have

$$
\begin{aligned}
\mathrm{CH}_{N}^{-p} & =V_{N} D_{N}^{-p} V_{N}^{-1}=\left(\left(V_{N}^{-1}\right)^{-1} D_{N}^{p} V_{N}^{-1}\right)^{-1} \\
& =\left(V_{N} D_{N}^{p} V_{N}^{-1}\right)^{-1}=\left(\mathrm{CH}_{N}^{p}\right)^{-1} .
\end{aligned}
$$

The proof is complete. 
TABLE 1: Discrete FrCOSHAD transform $\mathrm{CH}_{N}^{p}$ of several functions with $m=3$ and various fractional order $p$.

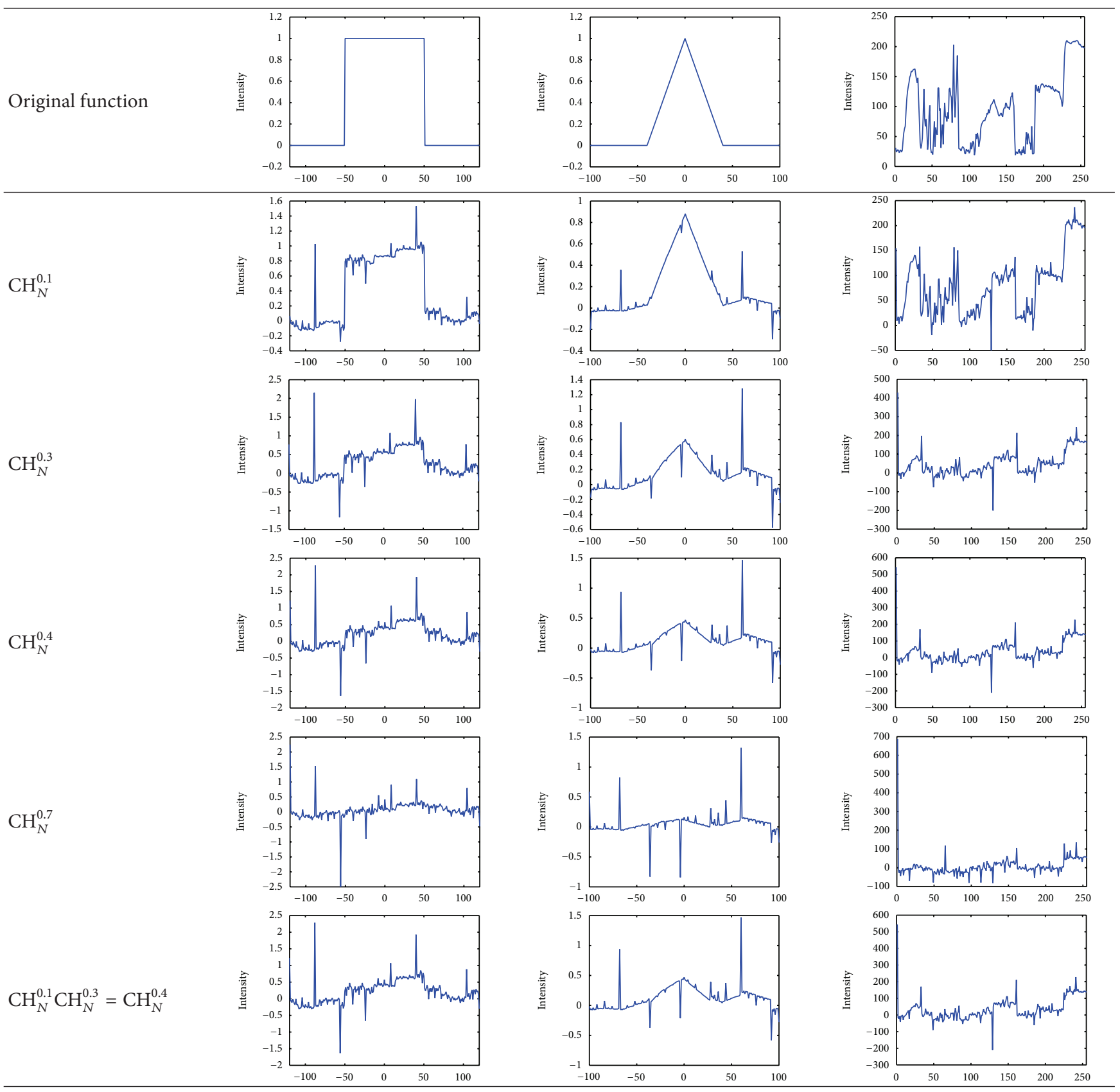

3.3. Quaternion Discrete Orthogonal Transform. Traditional methods process colour images by processing each colour channel separately and then summing the three individual outputs to derive the final result. Ell and Sangwine [19] were the first to use quaternions to encode colour images as vector fields. Motivated by their work, this paper defines the so-called Q-COSHAD and Q-FrCOSHAD transforms. We consider here only RGB colour images. The generalisation to other types of colour images is not difficult. Let $\mathbf{f}$ be an RGB image; the three channels of the image can be represented using the three components of a pure quaternion as follows:

$$
\mathbf{f}=\mathbf{f}_{R} \mathbf{i}+\mathbf{f}_{G} \mathbf{j}+\mathbf{f}_{B} \mathbf{k}
$$

where $\mathbf{f}_{R}, \mathbf{f}_{G}$, and $\mathbf{f}_{B}$ represent the red, green, and blue components of the colour image, respectively. This representation effectively equates the RGB colour cube to the righthanded coordinate frame imposed by the imaginary part of the quaternion space and treats the colour image as a whole rather than as separate components. As an example, we now introduce the definition of the right-side form of the Q-FrCOSHAD transform of a function with two variables. Similar methods can be used to obtain the Q-COSHAD transform matrices.

Definition 2. Given a quaternion root of $-1, \boldsymbol{\mu}$, for an $N \times N$ colour image $\mathbf{f}$, the right-side form of the forward 


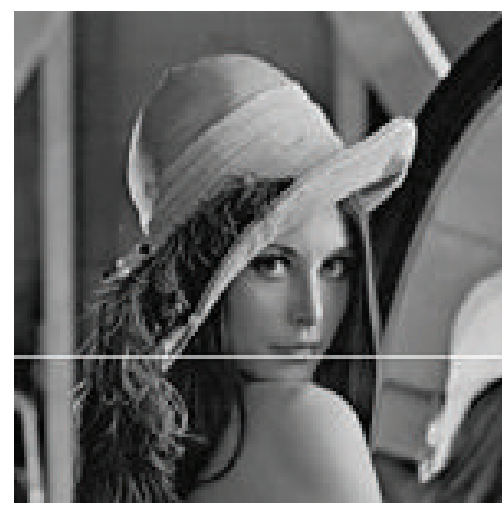

(a)

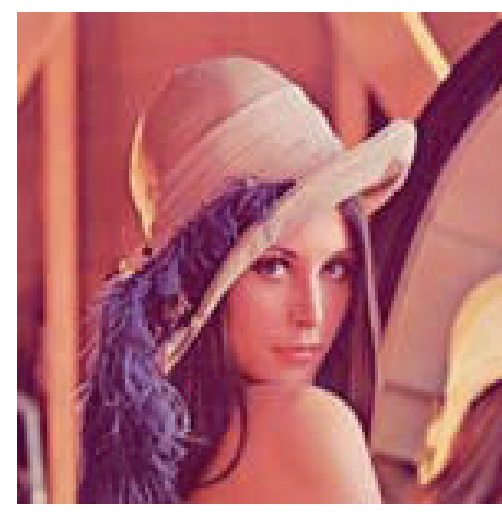

(b)

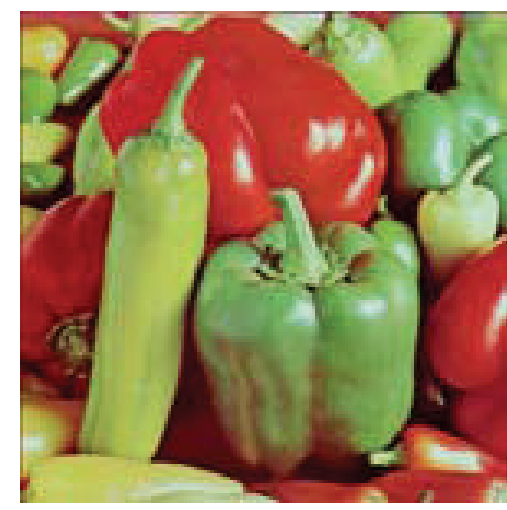

(c)

FIGURE 1: Test images from the USC-SIPI image databases: (a) line profile at row 180 of image Lena (gray); (b) Lena (Colour); (c) Peppers (Colour).

Q-FrCOSHAD transform of the image is defined using the matrix form as

$$
\begin{aligned}
\mathrm{QCH}_{N}^{p q}(\mathbf{f}) \_ & R \\
=( & \left(\left[\left(\frac{1}{\sqrt{N}}\right)^{p} \mathrm{CH}_{N}^{p}\right] \mathbf{f}_{R}\left[\left(\frac{1}{\sqrt{N}}\right)^{q} \mathrm{CH}_{N}^{q}\right]^{T}\right) \mathbf{i} \\
+ & \left(\left[\left(\frac{1}{\sqrt{N}}\right)^{p} \mathrm{CH}_{N}^{p}\right] \mathbf{f}_{G}\left[\left(\frac{1}{\sqrt{N}}\right)^{q} \mathrm{CH}_{N}^{q}\right]^{T}\right) \mathbf{j} \\
+ & \left.\left(\left[\left(\frac{1}{\sqrt{N}}\right)^{p} \mathrm{CH}_{N}^{p}\right] \mathbf{f}_{B}\left[\left(\frac{1}{\sqrt{N}}\right)^{q} \mathrm{CH}_{N}^{q}\right]^{T}\right) \mathbf{k}\right) \boldsymbol{\mu},
\end{aligned}
$$

where $\boldsymbol{\mu}=-(\mathbf{i}+\mathbf{j}+\mathbf{k}) / \sqrt{3}$ and $\boldsymbol{\mu}^{2}=\mathbf{i}^{2}=\mathbf{j}^{2}=\mathbf{k}^{2}=-1$. This choice corresponds to the single coloured line in RGB space, on which all three components are equal. Letting $\mathbf{F}_{R}, \mathbf{F}_{G}$, and $\mathbf{F}_{B}$ be the discrete FrCOSHAD transforms from (14) for the red, green, and blue components, respectively, of the colour image. Equation (22) can be rewritten as

$$
\begin{aligned}
\mathrm{QCH}_{N}^{p q}(\mathbf{f}) \_R \\
=\left(\mathbf{F}_{R} \mathbf{i}+\mathbf{F}_{G} \mathbf{j}+\mathbf{F}_{B} \mathbf{k}\right) \boldsymbol{\mu} \\
=-\frac{\left(\mathbf{F}_{R} \mathbf{i}+\mathbf{F}_{G} \mathbf{j}+\mathbf{F}_{B} \mathbf{k}\right)(\mathbf{i}+\mathbf{j}+\mathbf{k})}{\sqrt{3}} \\
=\mathbf{A}_{0}+\mathbf{i} \mathbf{A}_{1}+\mathbf{j} \mathbf{A}_{2}+\mathbf{k} \mathbf{A}_{3},
\end{aligned}
$$

where

$$
\begin{aligned}
& \mathbf{A}_{0}=\frac{1}{\sqrt{3}}\left(\mathbf{F}_{R}+\mathbf{F}_{G}+\mathbf{F}_{B}\right), \\
& \mathbf{A}_{1}=-\frac{1}{\sqrt{3}}\left(\mathbf{F}_{G}-\mathbf{F}_{B}\right),
\end{aligned}
$$

$$
\begin{aligned}
& \mathbf{A}_{2}=-\frac{1}{\sqrt{3}}\left(\mathbf{F}_{B}-\mathbf{F}_{R}\right), \\
& \mathbf{A}_{3}=-\frac{1}{\sqrt{3}}\left(\mathbf{F}_{R}-\mathbf{F}_{G}\right) .
\end{aligned}
$$

This paper defines the left-side forward Q-FrCOSHAD transform similarly by interchanging the positions of the colour image and the imaginary part $\boldsymbol{\mu}$.

Definition 3. Given a quaternion root of $-1, \boldsymbol{\mu}$, for an $N \times$ $N$ colour image $\mathbf{f}$, the left-side form of the forward QFrCOSHAD transform of the image is defined as

$$
\begin{aligned}
\mathrm{QCH}_{N}^{p q}(\mathbf{f}) & L \\
=\boldsymbol{\mu}( & \left(\left[\left(\frac{1}{\sqrt{N}}\right)^{p} \mathrm{CH}_{N}^{p}\right] \mathbf{f}_{R}\left[\left(\frac{1}{\sqrt{N}}\right)^{q} \mathrm{CH}_{N}^{q}\right]^{T}\right) \mathbf{i} \\
+ & \left(\left[\left(\frac{1}{\sqrt{N}}\right)^{p} \mathrm{CH}_{N}^{p}\right] \mathbf{f}_{G}\left[\left(\frac{1}{\sqrt{N}}\right)^{q} \mathrm{CH}_{N}^{q}\right]^{T}\right) \mathbf{j} \\
+ & \left.\left(\left[\left(\frac{1}{\sqrt{N}}\right)^{p} \mathrm{CH}_{N}^{p}\right] \mathbf{f}_{B}\left[\left(\frac{1}{\sqrt{N}}\right)^{q} \mathrm{CH}_{N}^{q}\right]^{T}\right) \mathbf{k}\right) .
\end{aligned}
$$

Corresponding to the Q-FrCOSHAD transform, there are two forms of the inverse Q-FrCOSHAD transform. The rightside inverse transform satisfies the following equations:

$$
\mathrm{IQCH}_{N}^{p q}-R
$$

$$
\begin{aligned}
=-( & {\left[\left(\frac{1}{\sqrt{N}}\right)^{-p} \mathrm{CH}_{N}^{-p}\right] \mathbf{A}_{0}\left[\left(\frac{1}{\sqrt{N}}\right)^{-q} \mathrm{CH}_{N}^{-q}\right]^{T} } \\
+ & \mathbf{i}\left[\left(\frac{1}{\sqrt{N}}\right)^{-p} \mathrm{CH}_{N}^{-p}\right] \mathbf{A}_{1}\left[\left(\frac{1}{\sqrt{N}}\right)^{-q} \mathrm{CH}_{N}^{-q}\right]^{T}
\end{aligned}
$$




$$
\begin{aligned}
&+ \mathbf{j}\left[\left(\frac{1}{\sqrt{N}}\right)^{-p} \mathrm{CH}_{N}^{-p}\right] \mathbf{A}_{2}\left[\left(\frac{1}{\sqrt{N}}\right)^{-q} \mathrm{CH}_{N}^{-q}\right]^{T} \\
&\left.+\mathbf{k}\left[\left(\frac{1}{\sqrt{N}}\right)^{-p} \mathrm{CH}_{N}^{-p}\right] \mathbf{A}_{3}\left[\left(\frac{1}{\sqrt{N}}\right)^{-q} \mathrm{CH}_{N}^{-q}\right]^{T}\right) \\
& \times \frac{(\mathbf{i}+\mathbf{j}+\mathbf{k})}{\sqrt{3}} \\
&=\mathbf{a}_{0}+\mathbf{i a}_{1}+\mathbf{j a}_{2}+\mathbf{k a}_{3},
\end{aligned}
$$

where

$$
\begin{aligned}
& \mathbf{a}_{0}=\frac{1}{\sqrt{3}}\left(\mathbf{f}_{\mathrm{A}_{1}}+\mathbf{f}_{\mathrm{A}_{2}}+\mathbf{f}_{\mathrm{A}_{3}}\right), \\
& \mathbf{a}_{1}=-\frac{1}{\sqrt{3}} \mathbf{i}\left(\mathbf{f}_{\mathrm{A}_{0}}+\mathbf{f}_{\mathrm{A}_{2}}-\mathbf{f}_{\mathrm{A}_{3}}\right), \\
& \mathbf{a}_{2}=-\frac{1}{\sqrt{3}} \mathbf{j}\left(\mathbf{f}_{\mathrm{A}_{0}}-\mathbf{f}_{\mathrm{A}_{1}}+\mathbf{f}_{\mathrm{A}_{3}}\right), \\
& \mathbf{a}_{3}=-\frac{1}{\sqrt{3}} \mathbf{k}\left(\mathbf{f}_{\mathrm{A}_{0}}+\mathbf{f}_{\mathrm{A}_{1}}-\mathbf{f}_{\mathrm{A}_{2}}\right),
\end{aligned}
$$

where $\mathbf{a}_{1}, \mathbf{a}_{2}$, and $\mathbf{a}_{3}$ are the three colour components of the reconstructed image, and $\mathbf{f}_{\mathrm{A}_{0}}, \mathbf{f}_{\mathrm{A}_{1}}, \mathbf{f}_{\mathrm{A}_{2}}$, and $\mathbf{f}_{\mathrm{A}_{3}}$ are inverse FrCOSHAD transforms from (15) with respect to $\mathbf{A}_{0}, \mathbf{A}_{1}$, $\mathbf{A}_{2}$, and $\mathbf{A}_{3}$, respectively. The left-side inverse transform is similarly defined as follows:

$$
\begin{aligned}
& \mathrm{IQCH}_{N}^{p q} L \\
& =-\frac{(\mathbf{i}+\mathbf{j}+\mathbf{k})}{\sqrt{3}} \\
& \times\left(\left[\left(\frac{1}{\sqrt{N}}\right)^{-p} \mathrm{CH}_{N}^{-p}\right] \mathbf{A}_{0}\left[\left(\frac{1}{\sqrt{N}}\right)^{-q} \mathrm{CH}_{N}^{-q}\right]^{T}\right. \\
& +\mathbf{i}\left[\left(\frac{1}{\sqrt{N}}\right)^{-p} \mathrm{CH}_{N}^{-p}\right] \mathbf{A}_{1}\left[\left(\frac{1}{\sqrt{N}}\right)^{-q} \mathrm{CH}_{N}^{-q}\right]^{T} \\
& +\mathbf{j}\left[\left(\frac{1}{\sqrt{N}}\right)^{-p} \mathrm{CH}_{N}^{-p}\right] \mathbf{A}_{2}\left[\left(\frac{1}{\sqrt{N}}\right)^{-q} \mathrm{CH}_{N}^{-q}\right]^{T} \\
& \left.+\mathbf{k}\left[\left(\frac{1}{\sqrt{N}}\right)^{-p} \mathrm{CH}_{N}^{-p}\right] \mathbf{A}_{3}\left[\left(\frac{1}{\sqrt{N}}\right)^{-q} \mathrm{CH}_{N}^{-q}\right]^{T}\right) .
\end{aligned}
$$

This study will use the right-side forms $\mathrm{QCH}_{N}^{p q} R$ and $\mathrm{IQCH}_{N}^{p q} R$ as the default $\mathrm{QCH}_{N}^{p q}$ and $\mathrm{IQCH}_{N}^{p q}$, respectively, unless stated otherwise. Both $\mathrm{QCH}_{N}^{p q}$ and $\mathrm{IQCH}_{N}^{p q}$ pairs are separable and, thus, may be evaluated with a $1 \mathrm{D}$ summation over the rows and the columns of the input array. We naturally wonder whether the additivity property also holds for $2 \mathrm{D}$ QFrCOSHAD transform. This experiment is conducted with a colour image "Lena" that contains $512 \times 512$ pixels, as shown in Figure 1(b). The results of the Q-FrCOSHAD transform with different values of $p$ and $q$ are depicted in Figure 2. The last column of Figure 2 shows that the inverse transform results are very close to the results of the original image. The first four columns in Figure 2 show the four components $\mathbf{A}_{0}, \mathbf{A}_{1}$, $\mathbf{A}_{2}$, and $\mathbf{A}_{3}$ in (24) of the Q-FrCOSHAD transform. The last row of Figure 2 indicates that the Q-FrCOSHAD transform with $p=0.1, q=0.1$, followed by an application of the QFrCOSHAD transform with $p=0.2, q=0.3$, is equivalent to the application of the transform with $p=0.3, q=0.4$. Hence, we can reach the following conclusions. Regardless of what the fraction orders $p$ and $q$ are, 2D Q-FrCOSHAD can accurately recover the colour images in the noise-free cases. In addition, similarly to the $1 \mathrm{D}$ FrCOSHAD transform case, fractional additivity also holds in the 2D Q-FrCOSHAD case. Finally, we notice that the large fractional orders $p$ and $q$ lead to a significant effect for spectral images. In other words, a spectral image with small fractional orders is closer than the original image before the transformation.

\section{COSHAD-Based IPRM Is Spectrally Convergent}

Spectral approximations have been widely implemented in the approximation of signals $[20,21]$. The kernel functions very commonly used in spectral partial sum approximations are Fourier, Cosine, Legendre, Chebyshev, and Gegenbauer, and so forth. Sometimes, nonclassical kernel functions have been proven to be very useful for specific purposes. This important reason leads us directly to discuss the possibility of representing a signal from a limited number of expansion coefficients of COSHAD/FrCOSHAD with high accuracy.

The overshoot of an approximation to a function near the discontinuity or the domain boundaries will occur for most approximations based on standard orthogonal expansions [22]. Many effective algorithms have been developed to overcome the overshoot and to improve the spectral accuracy [2325]. For obtaining IPRM in the discrete COSHAD domain, let us first introduce the explicit expression of the COSHAD kernel function as follows:

$$
\begin{aligned}
& \left.\operatorname{COSHAD}_{m}(n)\right] \\
& =\beta\left(k_{j}\right) \cos \left(\pi k_{j} \frac{2 p_{i}+1}{2^{m+1}}\right)(\mathrm{HAD})_{j \bmod 2^{n-m}+1, i \bmod 2^{n-m}+1}, \\
& \quad 0 \leq i, \quad j \leq N-1,
\end{aligned}
$$

with

$$
\begin{gathered}
N=2^{n}, \quad k_{j}=\left[\frac{j+1}{2^{n-m}}\right]-1, \quad p_{i}=\left[\frac{i+1}{2^{n-m}}\right]-1, \\
\beta\left(k_{j}\right)= \begin{cases}\frac{1}{\sqrt{2^{m}}}, \quad k_{j}=0, \\
\frac{1}{\sqrt{2^{m-1}}}, \quad k_{j}=1, \ldots, 2^{m}-1 .\end{cases}
\end{gathered}
$$

In the above, the symbol (HAD) $)_{a, b}$ represents the ath row and $b$ th column elements of the Hadamard kernel function, "mod" represents a modulo operation, and "[-]" represents 

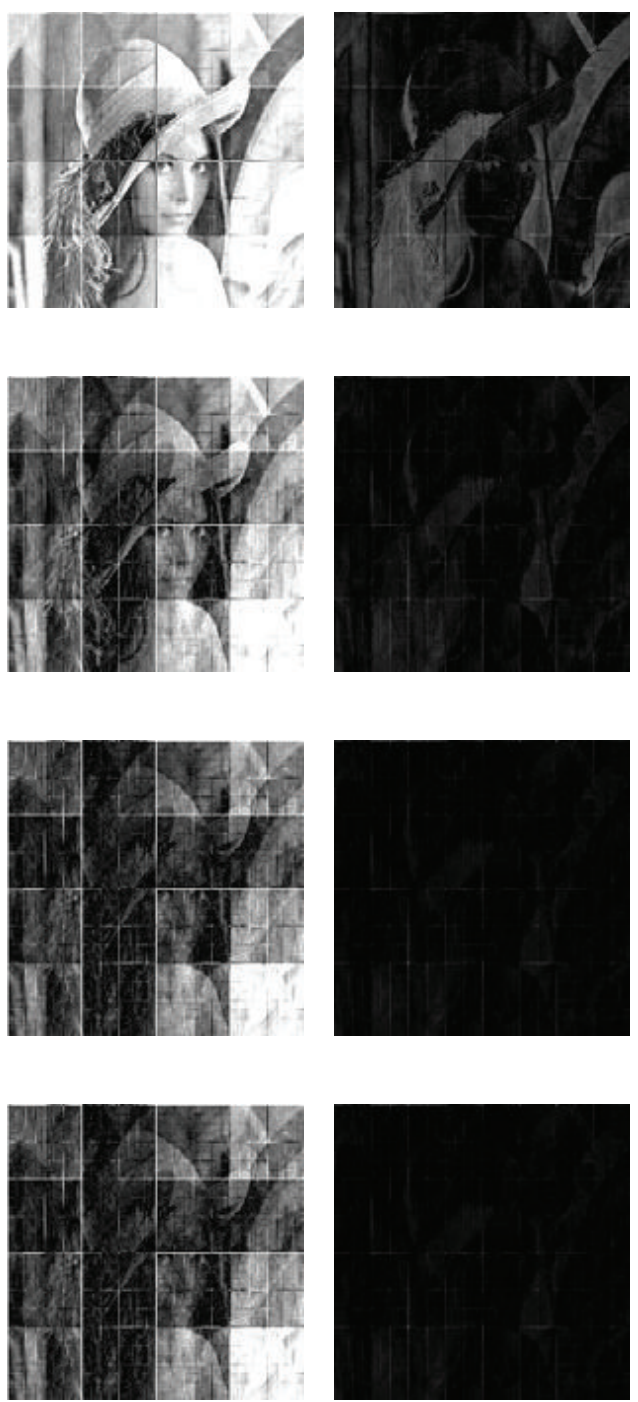
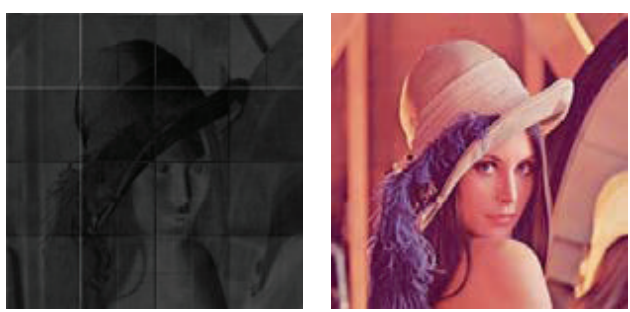

(a)
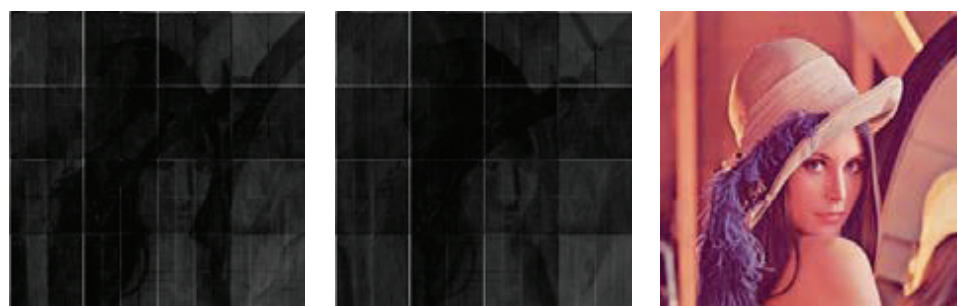

(b)
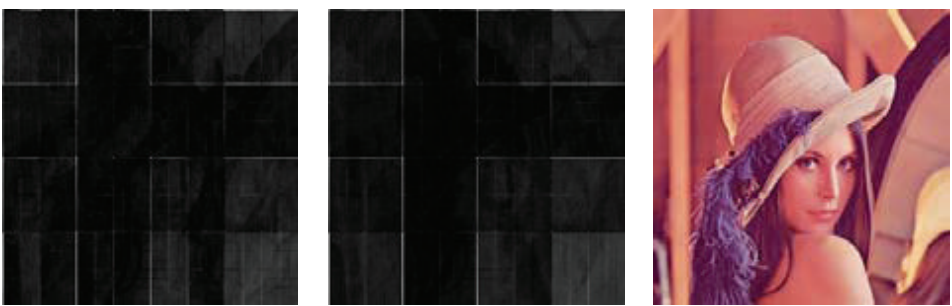

(c)
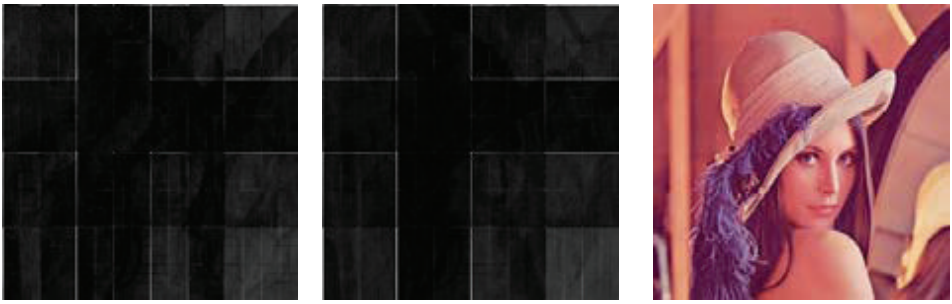

(d)

Figure 2: Discrete Q-FrCOSHAD transform $\mathrm{QCH}_{N}^{p q}(\mathbf{f})$ of colour image "Lena" ( $\left.m=2\right)$. Transform values from left to right are $\mathbf{A}_{0}, \mathbf{A}_{1}, \mathbf{A}_{2}$, and $\mathbf{A}_{3}$, and reconstructed images: (a) $p=0.1, q=0.1$; (b) $p=0.2, q=0.3$; (c) $p=0.3, q=0.4$; (d) $\mathrm{QCH}_{N}^{p_{1}, q_{1}}\left(\mathrm{QCH}_{N}^{p_{2}, q_{2}}(\mathbf{f})\right)$, $p_{1}=0.1$, $q_{1}=0.1, p_{2}=0.2, q_{2}=0.3$.

the ceiling function. Thus, applying (29), the COSHAD expansion of an unknown function $f\left(x_{i}\right)$ using standard inner product can be obtained; that is

$$
\begin{aligned}
& \widehat{f}_{k_{j}}=\left(f\left(x_{i}\right), \psi_{i, j}\left(p_{i}\right)\right)_{\text {COSHAD }} \\
& \equiv \frac{1}{\sqrt{N}} \sum_{i=0}^{N-1} f\left(x_{i}\right) \psi_{i, j}\left(p_{i}\right) \\
&=\frac{1}{\sqrt{N}} \sum_{i=0}^{N-1} \beta\left(k_{j}\right) f\left(x_{i}\right) \cos \left(\pi k_{j} \frac{2 p_{i}+1}{2^{m+1}}\right) \\
& \quad \times(\mathrm{HAD})_{j \bmod 2^{n-m}+1, i \bmod 2^{n-m}+1}
\end{aligned}
$$

where

$$
\begin{aligned}
\psi_{i, j}\left(p_{i}\right)= & \beta\left(k_{j}\right) \cos \left(\pi k_{j} \frac{2 p_{i}+1}{2^{m+1}}\right) \\
& \times(\mathrm{HAD})_{j \bmod 2^{n-m}+1, i \bmod 2^{n-m}+1} .
\end{aligned}
$$

Using (32), the truncated series expansion of a signal using only the first $N_{d}$ terms of the COSHAD coefficients is given by

$$
\begin{aligned}
& f^{N_{d}}\left(x_{i}\right)= \frac{1}{\sqrt{N}} \sum_{j=0}^{N_{d}-1}\left(f\left(x_{i}\right), \psi_{i, j}\left(p_{i}\right)\right)_{\operatorname{COSHAD}} \widetilde{\psi}_{i, j}\left(p_{i}\right) \\
&= \frac{1}{\sqrt{N}} \sum_{j=0}^{N_{d}-1} \widehat{f}_{k_{j}} \widetilde{\psi}_{i, j}\left(p_{i}\right) \\
&=\frac{1}{\sqrt{N}} \sum_{j=0}^{N_{d}-1} \beta\left(k_{j}\right) \widehat{f}_{k_{j}} \cos \left(\pi k_{j} \frac{2 p_{i}+1}{2^{m+1}}\right) \\
& \quad \times(\mathrm{HAD})_{i \bmod 2^{n-m}+1, j \bmod 2^{n-m}+1},
\end{aligned}
$$


where

$$
\begin{aligned}
\widetilde{\psi}_{i, j}\left(p_{i}\right)= & \beta\left(k_{j}\right) \cos \left(\pi k_{j} \frac{2 p_{i}+1}{2^{m+1}}\right) \\
& \times(\mathrm{HAD})_{i \bmod 2^{n-m}+1, j \bmod 2^{n-m}+1} .
\end{aligned}
$$

Assume that the original function $f\left(x_{i}\right)$ is analytic and this original function can be expanded in Gegenbauer orthogonal polynomials as follows:

$$
\begin{array}{r}
f_{u}\left(\bar{x}_{i}\right) \equiv f\left(x_{i}\right)=\sum_{l=0}^{u} g_{l} C_{l}^{\lambda}\left(\bar{x}_{i}\right), \\
0 \leq i \leq N-1,
\end{array}
$$

where $g_{l}$ are the Gegenbauer coefficients defined on the Gegenbauer transform domain

$$
g_{l}=\frac{1}{h_{l}^{\lambda}} \int_{-1}^{1}\left(1-\bar{x}_{i}^{2}\right)^{\lambda-1 / 2} f\left(\bar{x}_{i}\right) C_{l}^{\lambda}\left(\bar{x}_{i}\right) d \bar{x}_{i}
$$

with

$$
h_{l}^{\lambda}=\pi^{1 / 2} C_{l}^{\lambda}(1) \frac{\Gamma(\lambda+1 / 2)}{\Gamma(\lambda)(l+\lambda)} .
$$

$C_{l}^{\lambda}\left(\bar{x}_{i}\right)$ is the Gegenbauer polynomial of order $l$ with parameter $\lambda(\lambda>0)$, and $x_{i}$ is mapped as $\bar{x}_{i}=-1+2\left(x_{i}+1 / 2\right) / N$ to satisfy the domain of the Gegenbauer orthogonal polynomials. The purpose of the COSHAD-based IPRM method is to find a finite approximation $\widetilde{f}_{u}\left(\bar{x}_{i}\right)$ for the unknown function $f\left(x_{i}\right)$ as

$$
\tilde{f}_{u}\left(\bar{x}_{i}\right)=\sum_{l=0}^{u} \widetilde{g}_{l} C_{l}^{\lambda}\left(\bar{x}_{i}\right), \quad 0 \leq i \leq N-1 .
$$

So the error defined in the following:

$$
e\left(\bar{x}_{i}\right) \equiv \tilde{f}_{u}^{N_{d}}\left(\bar{x}_{i}\right)-f^{N_{d}}\left(x_{i}\right), \quad 0 \leq i \leq N-1
$$

is orthogonal to the COSHAD transform domain. That is,

$$
\left(e\left(\bar{x}_{i}\right), \psi_{i, j}\left(p_{i}\right)\right)_{\mathrm{COSHAD}}=0,
$$

where

$$
\begin{array}{r}
\tilde{f}_{u}^{N_{d}}\left(\bar{x}_{i}\right) \\
=\frac{1}{\sqrt{N}} \sum_{j=0}^{N_{d}-1}\left(\tilde{f}_{u}\left(\bar{x}_{i}\right), \psi_{i, j}\left(p_{i}\right)\right)_{\mathrm{COSHAD}} \widetilde{\psi}_{i, j}\left(p_{i}\right), \\
0 \leq i \leq N-1 .
\end{array}
$$

By substituting $f\left(x_{i}\right)$ in (32) with $\tilde{f}_{u}\left(\bar{x}_{i}\right)$ from (39), we have

$$
\begin{aligned}
& \widehat{f}_{k_{j}}= \frac{1}{\sqrt{N}} \sum_{i=0}^{N-1} \tilde{f}_{u}\left(\bar{x}_{i}\right) \psi_{i, j}\left(p_{i}\right) \\
&=\frac{1}{\sqrt{N}} \sum_{i=0}^{N-1} \beta\left(k_{j}\right) \tilde{f}_{u}\left(\bar{x}_{i}\right) \cos \left(\pi k_{j} \frac{2 p_{i}+1}{2^{m+1}}\right) \\
& \quad \times(\mathrm{HAD})_{j \bmod 2^{n-m}+1, i \bmod 2^{n-m}+1} \\
&=\frac{1}{\sqrt{N}} \sum_{i=0}^{N-1} \sum_{l=0}^{u} \beta\left(k_{j}\right) \widetilde{g}_{l} C_{l}^{\lambda}\left(\bar{x}_{i}\right) \cos \left(\pi k_{j} \frac{2 p_{i}+1}{2^{m+1}}\right) \\
& \quad \times(\mathrm{HAD})_{j \bmod 2^{n-m}+1, i \bmod 2^{n-m}+1} .
\end{aligned}
$$

By defining the matrix $\mathbf{W}$ as

$$
\mathbf{W}=\left[W_{k_{j} l}\right], \quad k_{j}=0, \ldots,\left[\frac{j+1}{2^{n-m}}\right]-1, l=0, \ldots, u,
$$

with matrix elements $W_{k_{j}}$ given by

$$
\begin{aligned}
& W_{k_{j} l}= \frac{1}{\sqrt{N}} \sum_{i=0}^{N-1} C_{l}^{\lambda}\left(\bar{x}_{i}\right) \psi_{i, j}\left(p_{i}\right) \\
&=\frac{1}{\sqrt{N}} \sum_{i=0}^{N-1} \beta\left(k_{j}\right) C_{l}^{\lambda}\left(\bar{x}_{i}\right) \cos \left(\pi k_{j} \frac{2 p_{i}+1}{2^{m+1}}\right) \\
& \quad \times(\mathrm{HAD})_{j \bmod 2^{n-m}+1, i \bmod 2^{n-m}+1} .
\end{aligned}
$$

Equation (43) can be rewritten as

$$
\widehat{f}_{k_{j}}=\sum_{l=0}^{u} \widetilde{g}_{l} W_{k_{j} l}
$$

Equation (46) can also be written in matrix form as

$$
\widehat{\mathbf{f}}=\mathbf{W} \widetilde{\mathbf{G}}
$$

where the vectors $\widetilde{\mathbf{G}}$ and $\widehat{\mathbf{f}}$ are

$$
\begin{aligned}
\widetilde{\mathbf{G}} & =\left[\widetilde{g}_{0}, \widetilde{g}_{1}, \ldots, \widetilde{g}_{u}\right], \\
\widehat{\mathbf{f}} & =\left[\widehat{f}_{0}, \widehat{f}_{1}, \ldots, \widehat{f}_{\left[N_{d} / 2^{n-m}\right]-1}\right],
\end{aligned}
$$

respectively. Under the constraint

$$
1+u \leq\left[\frac{N_{d}}{2^{n-m}}\right]
$$

we can obtain the following Gegenbauer coefficients $\widetilde{\mathbf{G}}$ through the pseudo-inversion of $\mathbf{W}$ by

$$
\widetilde{\mathbf{G}}=\mathbf{W}^{\dagger} \cdot \widehat{\mathbf{f}} .
$$

To prove the convergence of the proposed COSHADbased IPRM, we define the truncation error $e_{t}\left(\bar{x}_{i}\right)$ and the regularisation error $e_{r}\left(\bar{x}_{i}\right)$ as follows

$$
e_{t}\left(\bar{x}_{i}\right)=f_{u}\left(\bar{x}_{i}\right)-\tilde{f}_{u}\left(\bar{x}_{i}\right), \quad e_{r}\left(\bar{x}_{i}\right)=f\left(x_{i}\right)-f_{u}\left(\bar{x}_{i}\right) .
$$


Lemma 4. If $i$ satisfies $0 \leq i \leq N-1$, then

$$
\left(e_{t}\left(\bar{x}_{i}\right)+e_{r}\left(\bar{x}_{i}\right), \psi_{i, j}\left(p_{i}\right)\right)_{\mathrm{COSHAD}}=0 .
$$

Proof. According to (40)-(41), one can obviously obtain

$$
\left(\tilde{f}_{u}\left(\bar{x}_{i}\right)-f\left(x_{i}\right), \psi_{i, j}\left(p_{i}\right)\right)_{\mathrm{COSHAD}}=0
$$

Thus,

$$
\begin{gathered}
\left(-\tilde{f}_{u}\left(\bar{x}_{i}\right), \psi_{i, j}\left(p_{i}\right)\right)_{\mathrm{COSHAD}}+\left(f\left(x_{i}\right), \psi_{i, j}\left(p_{i}\right)\right)_{\mathrm{COSHAD}}=0 \\
\left(f_{u}\left(\bar{x}_{i}\right)-\tilde{f}_{u}\left(\bar{x}_{i}\right), \psi_{i, j}\left(p_{i}\right)\right)_{\mathrm{COSHAD}} \\
+\left(f\left(x_{i}\right)-f_{u}\left(\bar{x}_{i}\right), \psi_{i, j}\left(p_{i}\right)\right)_{\mathrm{COSHAD}}=0
\end{gathered}
$$

Combining (52) and (56), one has

$$
\left(e_{t}\left(\bar{x}_{i}\right), \psi_{i, j}\left(p_{i}\right)\right)_{\mathrm{COSHAD}}+\left(e_{r}\left(\bar{x}_{i}\right), \psi_{i, j}\left(p_{i}\right)\right)_{\mathrm{COSHAD}}=0 .
$$

Thus,

$$
\left(e_{t}\left(\bar{x}_{i}\right)+e_{r}\left(\bar{x}_{i}\right), \psi_{i, j}\left(p_{i}\right)\right)_{\mathrm{COSHAD}}=0 .
$$

The lemma is, thus, proven.

Lemma 5. Let $f\left(x_{i}\right)$ be an analytic function. Then, there exists a constant $A$ that is independent of $u$, so that the regularisation error function $e_{r}\left(\bar{x}_{i}\right)$ defined in (52) satisfies the following inequality:

$$
\max _{-1 \leq \bar{x}_{i} \leq 1}\left|e_{r}\left(\bar{x}_{i}\right)\right| \leq 2^{-u} A
$$

Because the IPRM is independent on the basis function [26], we can choose the Chebyshev polynomial $C_{l}^{\lambda}\left(\bar{x}_{i}\right)=$ $T_{l}\left(\bar{x}_{i}\right)$ in (37) with $\lambda=0$. Thus, the proof of Lemma 5 can easily be obtained by the same procedure as in [27].

Theorem 6 (spectral convergence). ID IPRM for the discrete COSHAD expansion is spectrally convergent.

Proof. By Lemmas 4 and 5, if $0 \leq i \leq N-1$,

$$
\begin{aligned}
& \left|\left(e_{t}\left(\bar{x}_{i}\right), \psi_{i, j}\left(p_{i}\right)\right)_{\mathrm{COSHAD}}\right| \\
& \quad=\left|\left(e_{r}\left(\bar{x}_{i}\right), \psi_{i, j}\left(p_{i}\right)\right)_{\mathrm{COSHAD}}\right| \\
& \quad \leq\left|\left(\max _{-1 \leq \bar{x}_{i} \leq 1}\left|e_{r}\left(\bar{x}_{i}\right)\right|, \psi_{i, j}\left(p_{i}\right)\right)_{\mathrm{COSHAD}}\right| \\
& \quad \leq\left|\left(2^{-u} A, \psi_{i, j}\left(p_{i}\right)\right)_{\mathrm{COSHAD}}\right| \\
& \quad=\mid \frac{1}{\sqrt{N}} \sum_{i=0}^{N-1} \beta\left(k_{j}\right) 2^{-u} A \cos \left(\pi k_{j} \frac{2 p_{i}+1}{2^{m+1}}\right)
\end{aligned}
$$

$$
\begin{aligned}
& \quad \times(\mathrm{HAD})_{j \bmod 2^{n-m}+1, i \bmod 2^{n-m}+1} \mid \\
& =\frac{1}{\sqrt{N}} \beta\left(k_{j}\right) 2^{-u} A\left|\sum_{i=0}^{N-1} \cos \left(\pi k_{j} \frac{2 p_{i}+1}{2^{m+1}}\right)\right| \\
& \leq \beta\left(k_{j}\right) 2^{-u} A \sqrt{N} .
\end{aligned}
$$

Let $e_{t}^{N_{d}}\left(\bar{x}_{i}\right)$ denote the COSHAD approximation of $e_{t}\left(\bar{x}_{i}\right)$. Using $u=N_{d}$,

$$
\begin{aligned}
& \max _{-1 \leq \bar{x}_{i} \leq 1}\left|e_{t}^{N_{d}}\left(\bar{x}_{i}\right)\right| \\
& =\max _{-1 \leq \bar{x}_{i} \leq 1}\left|\frac{1}{\sqrt{N}} \sum_{j=0}^{N_{d}-1}\left(e_{t}\left(\bar{x}_{i}\right), \psi_{i, j}\left(p_{i}\right)\right)_{\text {COSHAD }} \widetilde{\psi}_{i, j}\left(p_{i}\right)\right| \\
& \leq\left|\frac{1}{\sqrt{N}} \sum_{j=0}^{N_{d}-1}\left(\max _{-1 \leq \bar{x}_{i} \leq 1} e_{t}\left(\bar{x}_{i}\right), \psi_{i, j}\left(p_{i}\right)\right)_{\operatorname{COSHAD}} \widetilde{\psi}_{i, j}\left(p_{i}\right)\right| \\
& =\mid \frac{1}{\sqrt{N}} \sum_{j=0}^{N_{d}-1} \beta\left(k_{j}\right) \beta\left(k_{j}\right) 2^{-u} A \cdot \sqrt{N} \cos \left(\pi k_{j} \frac{2 p_{i}+1}{2^{m+1}}\right) \\
& =\beta^{2}\left(k_{j}\right) 2^{-u} A\left|\sum_{j=0}^{N_{d}-1} \cos \left(\pi k_{j} \frac{2 p_{i}+1}{2^{m+1}}\right)\right| \\
& \leq \beta^{2}\left(k_{j}\right) 2^{-u} A \cdot N_{d} .
\end{aligned}
$$

Therefore, with (31), we have

$$
\max _{-1 \leq \bar{x}_{i} \leq 1}\left|e_{t}^{N_{d}}\left(\bar{x}_{i}\right)\right| \leq \begin{cases}2^{1-m} A q^{u+1}(u), & \text { for } k_{j}=0, \\ 2^{2-m} A q^{u+1}(u), & \text { for } k_{j} \neq 0,\end{cases}
$$

with

$$
q(u)=\frac{1}{2}(u+1)^{1 / u+1}
$$

Due to $q(u)<1$, for any number $u>0$, and $2^{2-m} \leq 1$, for $0 \leq m \leq n$, therefore,

$$
\max _{-1 \leq \bar{x}_{i} \leq 1}\left|e_{t}^{N_{d}}\left(\bar{x}_{i}\right)\right| \leq A q^{u+1}(u) .
$$

Theorem 6 is, thus, proven. Similar methods could be adapted to prove the spectral convergence of $1 \mathrm{D}$ FrCOSHAD-based IPRM without difficulty.

\section{Experimental Results}

5.1. Energy Compaction. The purpose of this subsection is to evaluate the coding efficiency of the proposed FrCOSHAD 


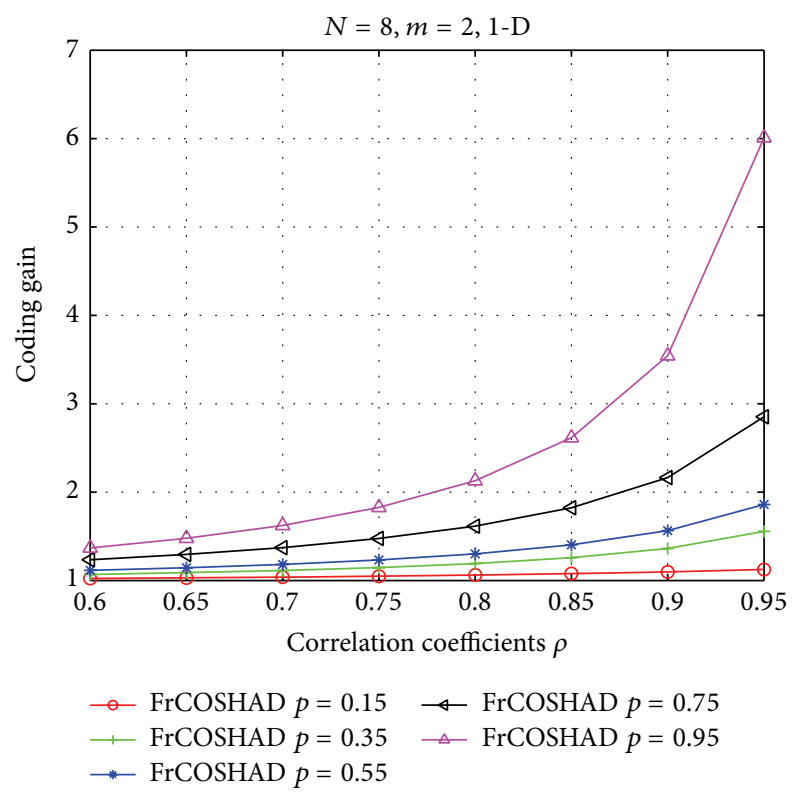

(a)

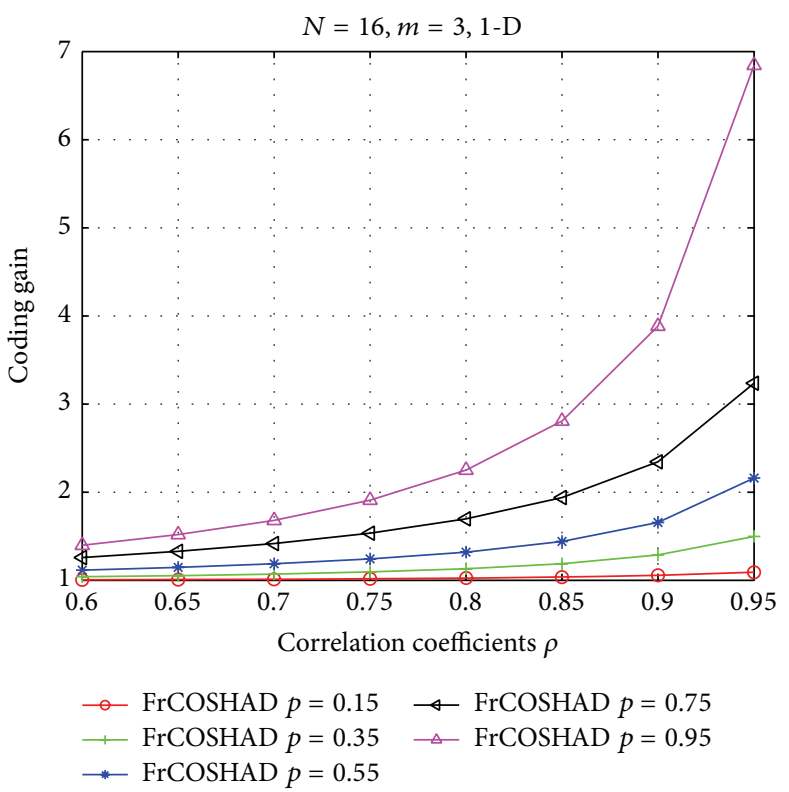

(b)

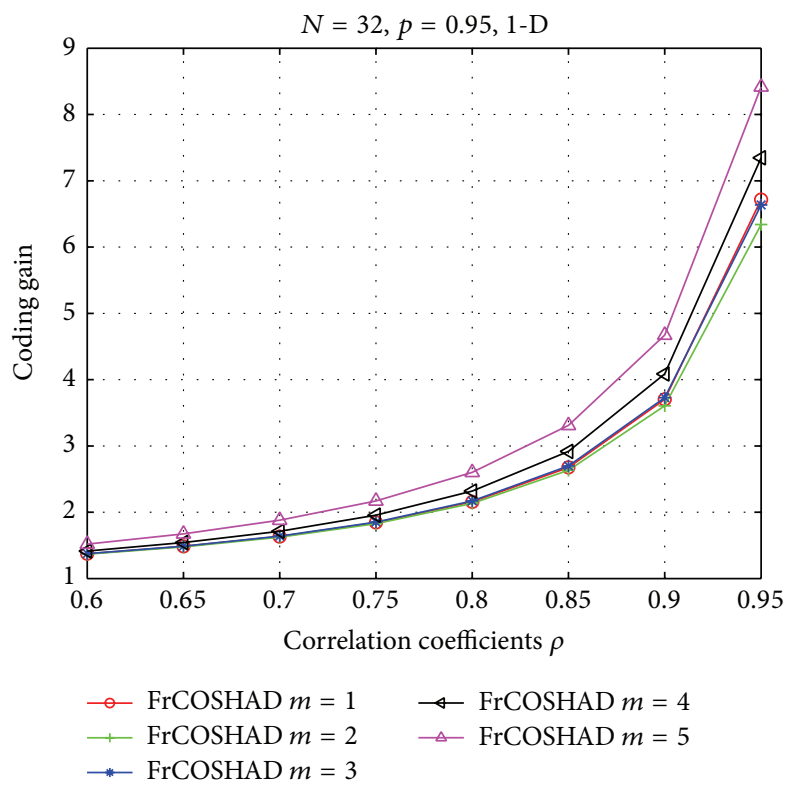

(c)

FIGURE 3: Influence of parameter $p$ and $m$ on the coding gain; (a) $N=8, m=2$; (b) $N=16, m=3$; (c) $N=32, p=0.95$.

transforms and to compare them with the other transforms. The coding gain [28] of the $1 \mathrm{D}$ and $2 \mathrm{D}$ cases will be investigated in this subsection. As mentioned above, understanding whether parameters $m$ and $p$ have an important influence over the coding gain. We first examine the fractional order $p$ with two examples where $m=2$ and 3 shown in Figures 3(a) and 3(b), respectively. This figure shows that the higher fractional order $p$ would generate a greater coding gain.

In the following experiment, we discuss the influence of parameter $m$ on the energy compaction. According to the constraints imposed on the parameters $m, n, N$, indicated by (1) and (2), we have systematically chosen the parameters as $N=32$ in this experiment where the value of fractional order $p$ is fixed at 0.95 , and only parameter $m$ is adjustable. The plots of coding gain are depicted in Figure 3(c). From this figure, the fifth choice $(m=5)$ gives the highest coding gain among all of the test cases. In contrast to fractional order $p$, parameter $m$ does not have as obvious an impact on the energy compaction. Only a limited decrease of the coding gain can be observed to respond to the decrease of parameter $m$.

Based on the above test results, we found that the energy compactions of the FrCOSHAD kernel matrix are significantly affected by order $p$ and are moderately affected by $m$. 

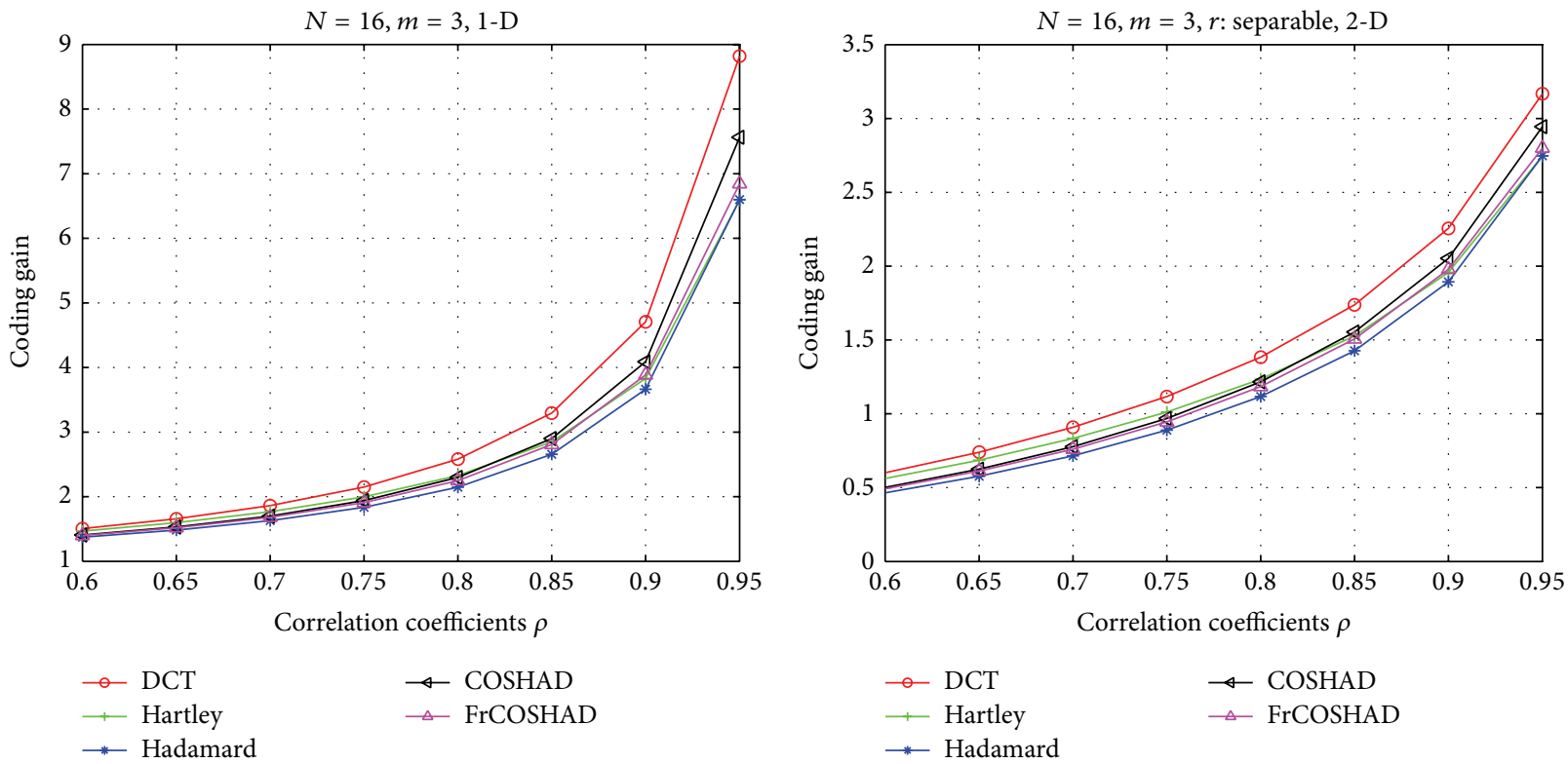

(a)

(b)

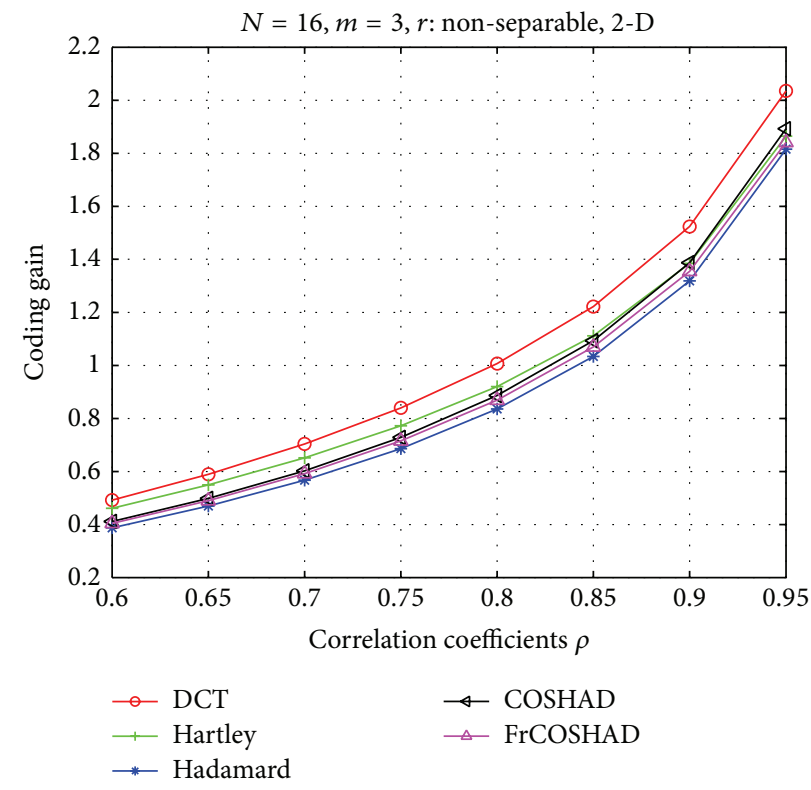

(c)

Figure 4: Comparison of the DCT, DHT, Hadamard, COSHAD, and FrCOSHAD transforms in terms of coding gain, (a) $N=16, m=3,1 \mathrm{D}$; (b) $N=16, m=3$, $r$ Separable, 2D; (c) $N=16, m=3, r$ : Non-separable, $2 \mathrm{D}$.

This result can be explained because the smaller the value offractional order $p$, the less the effect of the FrCOSHAD transform. The FrCOSHAD kernel function has not fully played a role in the process of transformation. So far as parameter $m$ is concerned, the choice of a relatively large $m$ corresponding to the case where the emphasis of the kernel function is the DCT properties rather than the Hadamard properties gives a high coding gain. Comparing the DCT and the Hadamard transforms, the DCT transform has a strong energy of compaction property, a well-known fact. Therefore, this is the main reason why we use such a choice of parameters $p=0.95$ and, as far as possible, big parameter $m$ for compressing the representations of images.

For comparison purposes, we calculated the coding gain with $N=16$ for the several transforms while correlation coefficient $\rho$ is varied from 0.6 to 0.95 . Figure 4 (a) show that the energy compaction capabilities from high to low in order is DCT, COSHAD, FrCOSHAD, DHT, and the Hadamard transforms. The fractional order of the FrCOSHAD is $p=$ 0.95 in this experiment.

A comparison of the results in terms of the $2 \mathrm{D}$ coding gains between the traditional 2D transforms (DCT, DHT, 

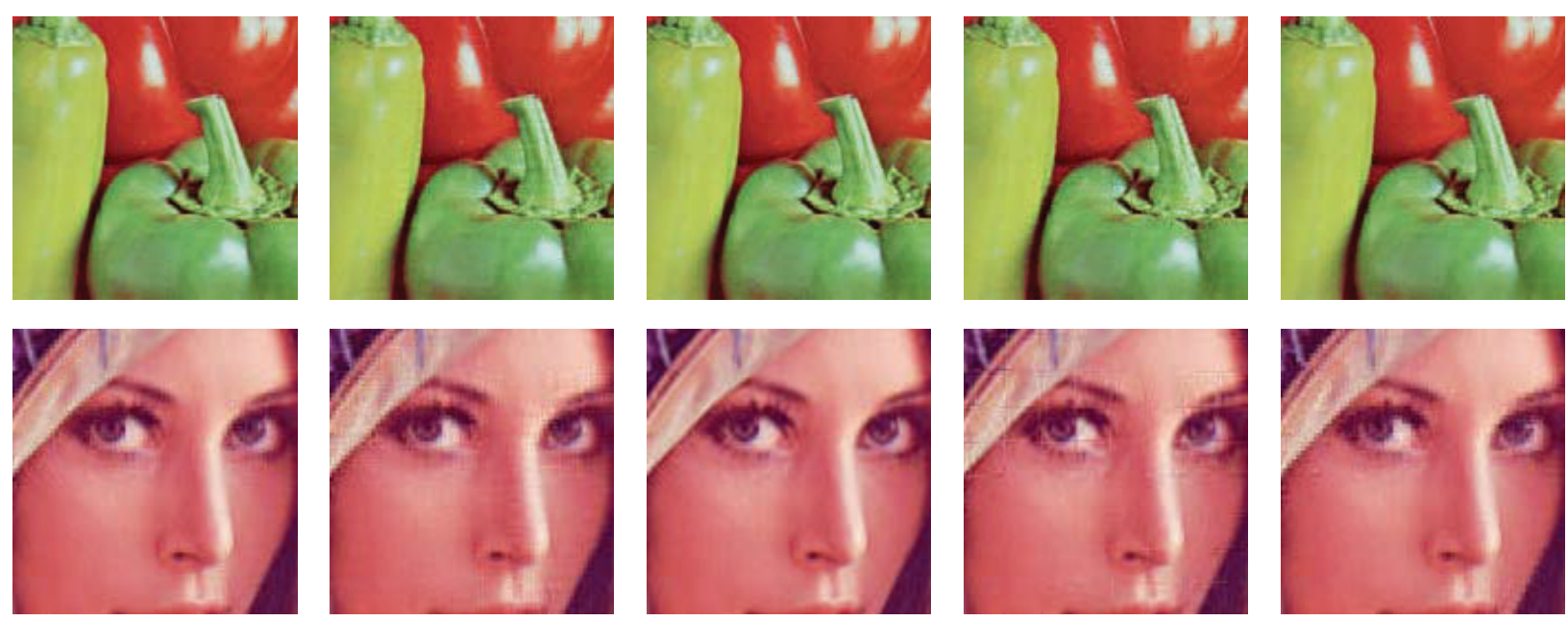

(a)

(b)

(c)

(d)

(e)

FIgURE 5: Local part of reconstructed images (block $=16 \times 16, \mathrm{Cr}$ is $1: 8$ ); (a) Q-COSHAD, $m=3$; (b) Q-FrCOSHAD, $m=3, p=0.95$, $q=0.95$; (c) Q-DCT; (d) Q-DHT; (e) Q-Hadamard.

and Hadamard) and the proposed transforms as $\rho$ increases is shown in Figures 4(b) and 4(c). The block sizes chosen for the experiment were $16 \times 16$. Regardless of whether the covariance $r$ is separable, the DCT transform consistently presents high coding gains in comparison with all of the other transforms. Both COSHAD and FrCOSHAD transforms had a higher coding efficiency than DHT and Hadamard transforms. The Hadamard transform yields poorer performance than all of the other transforms.

5.2. Applications of the Proposed Transforms to Colour Image Compression. In this subsection, we will compare the reconstructed results from the proposed transforms against QDCT [29], Q-DHT, and Q-Hadamard. The purpose is to confirm the theoretical analysis regarding the energy compaction. The experiments were conducted on two colour test images (size: $512 \times 512$ ) shown in Figures 1(b) and 1(c). We performed all simulations for these images within Matlab 7.11.0 (2010b) under Window environment on an Intel(R) Core(TM) i5 CPU $2.67 \mathrm{GHz}$ processor with $3 \mathrm{~GB}$ RAM.

Test images are first divided into equal-sized blocks, and each block is then transformed by using the kernel functions. Consequently, we arrange the absolute value of all of the transform coefficients in downward order and take the most significant part according to compression ratio $(\mathrm{Cr})$ to get a recovered image. The universal image quality index (UQI) [30] values of the reconstructed images with respect to the original images "Lena" and "Pepper" are listed in Tables 2 and 3, where $B$ denotes the block size used in experiment. According to the theoretical analysis of coding gain illustrated above, the fractional orders in the $2 \mathrm{D}$ FrCOSHAD transform were chosen as $p=0.95, q=0.95$. Tables 2 and 3 show that the qualities of the Q-DCT transform are overall higher than the qualities of the other transforms when $\mathrm{Cr}$ is $1: 2$ or $1: 8$. The reconstructed images using the proposed transform with $m=3$ perform not only better than the Q-COSHAD/FrCOSHAD with $m=2$ but also better than

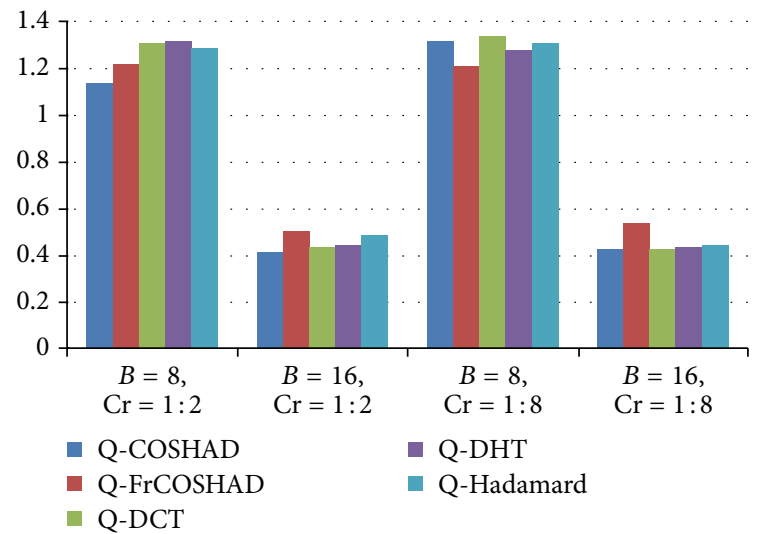

FIGURE 6: Comparison of time (s) required for reconstruction of colour image "Pepper".

the Q-DHT and the Q-Hadamard transforms. We think this is because the effect of the DCT on the FrCOSHAD method with $m=3$ is more obvious because the DCT has nearly optimal properties compared with the statistically optimal $\mathrm{K}-\mathrm{L}$ transform. The Q-Hadamard transform gives the worst reconstructions for all of the test images. Figure 5 shows that the images we cut out from the reconstructed images have no manipulation of any sort, only for viewing more clearly. In spite of having the same $B, C r$, and $m$, the reconstructed images are quite different: the Q-DCT, the Q-COSHAD, and the Q-FrCOSHAD transforms produced good results.

The computation times required for reconstruction of colour image "Pepper" are listed in Figure 6. Because of the simplicity of the block-based strategy, these algorithms can produce fast reconstructions along with relatively good visual qualities. In this test, $m=3$ is chosen for the Q-COSHAD/QFrCOSHAD transforms. However, the FrCOSHAD has a moderately computational burden for large block size due to the complexity of the algorithm. Similar results will be 
TABle 2: Performance comparison between the proposed transforms and the known algorithms for image "Lena".

\begin{tabular}{|c|c|c|c|c|c|c|c|c|}
\hline \multirow{2}{*}{ Methods } & \multicolumn{2}{|c|}{$B=8, \mathrm{Cr}=1: 2$} & \multicolumn{2}{|c|}{$B=16, \mathrm{Cr}=1: 2$} & \multicolumn{2}{|c|}{$B=8, \mathrm{Cr}=1: 8$} & \multicolumn{2}{|c|}{$B=16, \mathrm{Cr}=1: 8$} \\
\hline & $m=2$ & $m=3$ & $m=2$ & $m=3$ & $m=2$ & $m=3$ & $m=2$ & $m=3$ \\
\hline Q-COSHAD & 0.9778 & 0.9801 & 0.9866 & 0.9875 & 0.7974 & 0.8160 & 0.8814 & 0.8832 \\
\hline Q-FrCOSHAD & 0.9761 & 0.9768 & 0.9861 & 0.9869 & 0.7891 & 0.7926 & 0.8781 & 0.8798 \\
\hline Q-DCT & \multicolumn{2}{|c|}{0.9803} & \multicolumn{2}{|c|}{0.9887} & \multicolumn{2}{|c|}{0.8162} & \multicolumn{2}{|c|}{0.8975} \\
\hline Q-DHT & \multicolumn{2}{|c|}{0.9754} & \multicolumn{2}{|c|}{0.9859} & \multicolumn{2}{|c|}{0.7881} & \multicolumn{2}{|c|}{0.8766} \\
\hline Q-Hadamard & \multicolumn{2}{|c|}{0.9751} & \multicolumn{2}{|c|}{0.9853} & \multicolumn{2}{|c|}{0.7811} & \multicolumn{2}{|c|}{0.8720} \\
\hline
\end{tabular}

TABLE 3: Performance comparison between the proposed transforms and the known algorithms for image "Peppers".

\begin{tabular}{lccccccc}
\hline \multirow{2}{*}{ Methods } & \multicolumn{2}{c}{$B=8, \mathrm{Cr}=1: 2$} & \multicolumn{2}{c}{$B=16, \mathrm{Cr}=1: 2$} & \multicolumn{2}{c}{$B=8, \mathrm{Cr}=1: 8$} & \multicolumn{2}{c}{$B=16, \mathrm{Cr}=1: 8$} \\
& $m=2$ & $m=3$ & $m=2$ & $m=3$ & $m=2$ & $m=3$ & $m=2$ \\
\hline Q-COSHAD & 0.9696 & 0.9723 & 0.9845 & 0.9850 & 0.7786 & 0.7882 & 0.8715 \\
Q-FrCOSHAD & 0.9683 & 0.9694 & 0.9835 & 0.9849 & 0.7608 & 0.7685 & 0.8705 \\
Q-DCT & \multicolumn{2}{c}{0.9773} & \multicolumn{2}{c}{0.9865} & 0.7892 & 0.8723 \\
Q-DHT & \multicolumn{2}{c}{0.9678} & \multicolumn{2}{c}{0.9834} & 0.7560 & 0.8906 \\
Q-Hadamard & \multicolumn{2}{c}{0.9655} & 0.9831 & 0.7534 & 0.8698 \\
\hline
\end{tabular}

TABLe 4: Performance comparison between the proposed transforms and the known algorithms for image "Peppers" with different noise (PSNR in $\mathrm{dB}$ ).

\begin{tabular}{lcclc}
\hline \multirow{2}{*}{ Methods } & \multicolumn{2}{c}{$\begin{array}{c}\text { Salt \& Pepper } \\
\text { noise }(5 \%)\end{array}$} & \multicolumn{2}{c}{$\begin{array}{c}\text { Gaussian white noise } \\
\text { (mean: 0, variance: 0.01) }\end{array}$} \\
& $\mathrm{Cr}=1: 2$ & $\mathrm{Cr}=1: 4$ & $\mathrm{Cr}=1: 2$ & $\mathrm{Cr}=1: 4$ \\
\hline Q-COSHAD & 28.8986 & 22.9961 & 30.9782 & 25.0543 \\
Q-FrCOSHAD & 28.8880 & 22.9271 & 30.8836 & 24.9212 \\
Q-DCT & 28.9382 & 23.0320 & 31.0688 & 25.1468 \\
Q-DHT & 28.6947 & 22.5261 & 30.6381 & 24.7017 \\
Q-Hadamard & 28.3943 & 22.2529 & 30.3260 & 24.2723 \\
\hline
\end{tabular}

obtained with all other choices of the parameters $p, q$, and $m$. We omit discussing them, considering the space limit.

Generally speaking, the image representation quality can be severely affected by image noise. For the sake of evaluating the robustness of the proposed transforms against the negative effects of different types of noise, two experiments were conducted using noise-contaminated images. The first type of noise is the salt and pepper noise (5\%), while the second type of noise is the Gaussian white noise with zero mean and variance 0.01 . We conducted a few comparisons between the proposed approaches and the other schemes. The visual quality of the reconstructed image "Pepper" is measured using PSNR demonstrated in Table 4. Compared with the Q-DHT and the Q-Hadamard transforms, both the Q-COSHAD and the Q-FrCOSHAD produce a slightly higher PSNR under the same conditions. However, the QDCT produces the highest PSNR of the transforms in all cases with the presence of either Gaussian noise or salt and pepper noise.

In the following experiment, we evaluate the influence of $p$ and $q$ over the proposed Q-FrCOSHAD transform for the case of noise. Gaussian white noises with zero mean and variance 0.01 are taken into account in this test. Figure 7

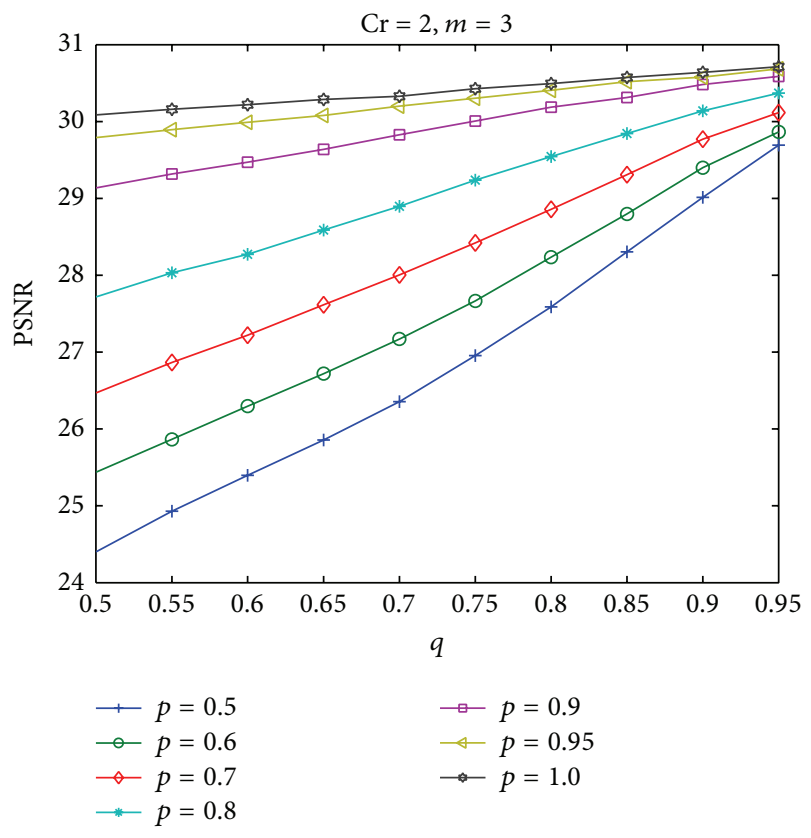

FIGURE 7: Reconstruction error of the colour image "Pepper" with Gaussian white noise (mean: 0, variance: 0.01) using FrCOSHAD transform (PSNR in $\mathrm{dB})$.

shows the corresponding PSNR values when $p$ and $q$ vary from 0.5 up to 1 . This figure shows that with the increase of fractional orders $p$ and $q$, the values of PSNR tend to rise gradually as expected. This example also proves once again that the effectiveness of the FrCOSHAD is severely affected by the fractional order: relatively large $p$ and $q$ leads to much better reconstructed results.

5.3. Local Representation by the IPRM. In this subsection, we attach three numerical examples that verify the accuracy of the proposed IPRM in the multi-intervals. Without loss of 


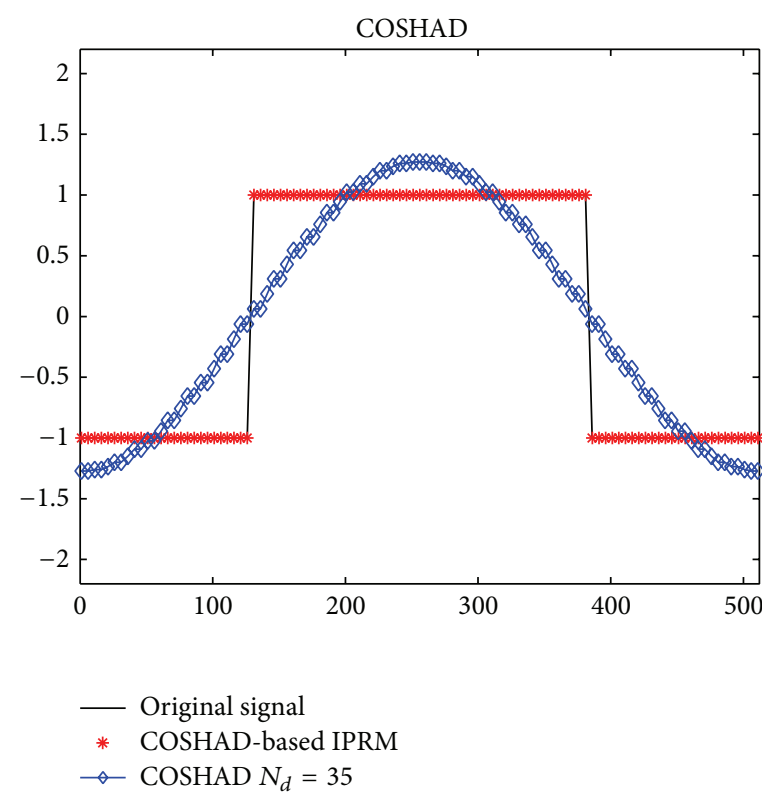

(a)

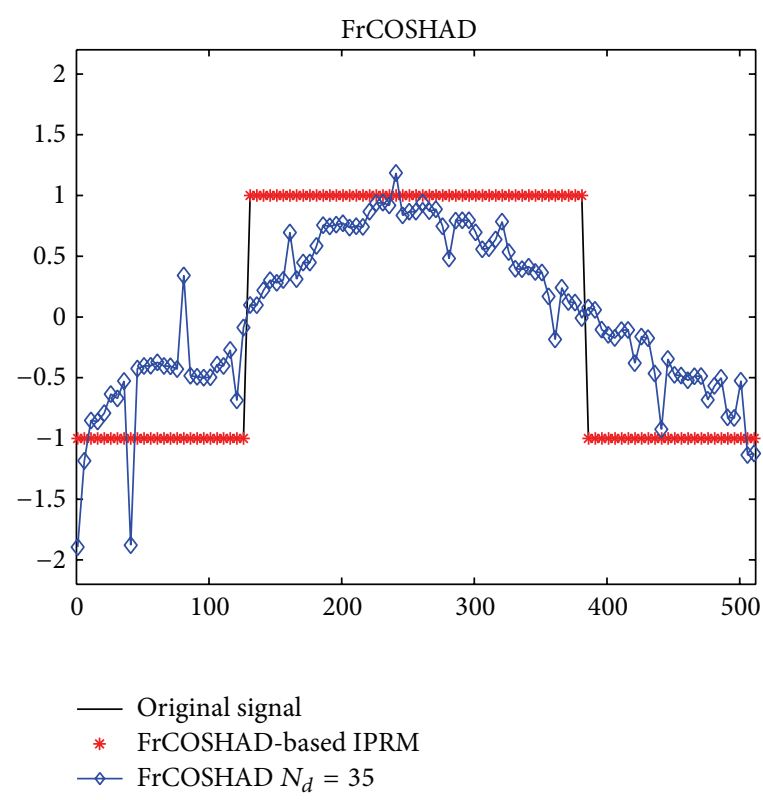

(b)

FIgURE 8: The function $f_{1}\left(x_{i}\right)$ and its approximation using the IPRM; (a) COSHAD-based IPRM; (b) FrCOSHAD-based IPRM.

generality, we first consider a window function $f_{1}\left(x_{i}\right)$ with length $N=512$ on the three subintervals depicted in Figure 8

$$
f_{1}\left(x_{i}\right)= \begin{cases}-1, & 0<x_{i} \leq 128 \\ 1, & 128<x_{i} \leq 384 \\ -1, & 384<x_{i} \leq 512\end{cases}
$$

In fact, the generalisation to more than three domains (or one axis) is also straightforward. If the length of signal $N$ is not a power of two, zero padding is needed to meet the constraint of the COSHAD kernel matrix (see (1) and (2)). In Figure 8, we show the original function $f_{1}\left(x_{i}\right)$ in the solid black line, the approximation through the COSHAD/FrCOSHAD partial sum with $N_{d}=35$ in the square dashed line. The COSHAD/FrCOSHAD partial sum approximation is obviously not accurate.

Next, we implement the proposed FrCOSHAD-based IPRM method in the following way. The original function $f_{1}\left(x_{i}\right)$ is first split into three pieces and solved for the smoother parts of the function in each small interval, and then the approximations are glued together to recover the original function $f_{1}\left(x_{i}\right)$ in the full interval. Parameter $\lambda$ of the Gegenbauer polynomials was fixed as 0.5 in all numerical examples. We get explicitly the approximate Gegenbauer coefficient $\widetilde{g}_{l}$, defined in (51) as follows.

For the left interval: $N_{d}=3, m=2, u=4$, and $p=0.4$ :

$$
\widetilde{g}_{l}=\left[\begin{array}{c}
-1.000000+8.444147 e-16 i \\
4.440892 e-16+9.271152 e-16 i \\
1.776356 e-15-3.192801 e-15 i
\end{array}\right] .
$$

For the middle interval: $N_{d}=4, m=3, u=3$, and $p=0.3$ :

$$
\widetilde{g}_{l}=\left[\begin{array}{c}
1.000000-6.327912 e-14 i \\
1.774664 e-29+6.375685 e-14 i \\
1.776356 e-15+6.149198 e-14 i \\
-3.552713 e-15-7.643780 e-14 i
\end{array}\right] .
$$

For the right interval: $N_{d}=5, m=2, u=4$, and $p=0.3$ :

$$
\tilde{g}_{l}=\left[\begin{array}{c}
-0.999999-5.210306 e-15 i \\
2.664535 e-15+4.001639 e-15 i \\
-1.776356 e-15+1.075126 e-14 i
\end{array}\right] .
$$

Once the approximate Gegenbauer coefficient $\widetilde{g}_{l}$ is obtained, one can compute the approximation to $f_{1}\left(x_{i}\right)$ by directly summing by using (39). If we let $\tilde{f}_{u}^{L}\left(\bar{x}_{i}\right), \tilde{f}_{u}^{M}\left(\bar{x}_{i}\right)$, and $\tilde{f}_{u}^{R}\left(\bar{x}_{i}\right)$ note the finite approximation for function $f_{1}\left(x_{i}\right)$ in the three subintervals, respectively, we can obtain the following approximation:

$$
\begin{aligned}
\tilde{f}_{u}^{L}\left(\bar{x}_{i}\right) \\
=\left(-1.000000+i 2.440815 e^{-15}\right) \\
\quad+\left(4.440892 e^{-16}+i 9.271153 e^{-16}\right) x \\
\quad+\left(2.664536 e^{-15}-i 4.789202 e^{-15}\right) x^{2},
\end{aligned}
$$


TABLE 5: $L_{\infty}$ error in a logarithmic scale, $N_{d}^{L}=3, N_{d}^{M}=4, N_{d}^{R}=4, u^{L}=2, u^{M}=3, u^{R}=3$.

\begin{tabular}{|c|c|c|c|c|c|}
\hline & Methods & $p^{L}$ & $p^{M}$ & $p^{R}$ & Error \\
\hline \multirow{5}{*}{$m^{L}=6, m^{M}=3, m^{R}=3$} & \multirow{4}{*}{ FrCOSHAD } & 0.6 & 0.4 & 0.5 & $3.9968 e-015$ \\
\hline & & 0.4 & 0.4 & 0.8 & $3.7748 e-015$ \\
\hline & & 0.2 & 0.6 & 0.2 & $1.2434 e-014$ \\
\hline & & 0.2 & 0.8 & 0.3 & $2.6423 e-014$ \\
\hline & COSHAD & 1 & 1 & 1 & $3.1240 e-014$ \\
\hline \multirow{5}{*}{$m^{L}=3, m^{M}=4, m^{R}=2$} & \multirow{4}{*}{ FrCOSHAD } & 0.6 & 0.4 & 0.5 & $5.4401 e-015$ \\
\hline & & 0.4 & 0.4 & 0.8 & $5.2382 e-015$ \\
\hline & & 0.2 & 0.6 & 0.2 & $2.4425 e-015$ \\
\hline & & 0.2 & 0.8 & 0.3 & $1.4433 e-015$ \\
\hline & COSHAD & 1 & 1 & 1 & $5.1098 e-014$ \\
\hline
\end{tabular}

$$
\begin{aligned}
\tilde{f}_{u}^{M}\left(\bar{x}_{i}\right) & \\
= & \left(1.000000-i 9.402512 e^{-14}\right) \\
& +\left(5.329071 e^{-15}+i 1.784136 e^{-13}\right) x \\
& +\left(2.664536 e^{-15}+i 9.223797 e^{-14}\right) x^{2} \\
& +\left(-8.881785 e^{-15}-i 1.910945 e^{-14}\right) x^{3} \\
\widetilde{f}_{u}^{R}\left(\bar{x}_{i}\right) & \\
= & \left(-0.999999-i 1.058594 e^{-14}\right) \\
& +\left(2.664535 e^{-15}+i 4.001639 e^{-15}\right) x \\
& +\left(-2.664536 e^{-15}+i 1.612690 e^{-14}\right) x^{2} .
\end{aligned}
$$

Similarly, using all of these parameters and only setting fraction order $p=1$, the approximation to $f_{1}\left(x_{i}\right)$ using the IPRM in the discrete COSHAD domain can be computed as follows:

$$
\begin{gathered}
\widetilde{f}_{u}^{L}\left(\bar{x}_{i}\right)=-1.000000+1.033642 e^{-20} x-5.787818 e^{-14} x^{2}, \\
\widetilde{f}_{u}^{M}\left(\bar{x}_{i}\right)=1.000000+6.444668 e^{-18} x \\
+2.468410 e^{-16} x^{2}-6.450610 e^{-18} x^{3}, \\
\widetilde{f}_{u}^{R}\left(\bar{x}_{i}\right)=-1.000000+2.427593 e^{-19} x+2.602084 e^{-18} x^{2} .
\end{gathered}
$$

Using the present methods, the solutions are obtained for three subintervals shown in Figure 8. The present strategies clearly provide an exact numerical solution rather than that obtained in the approximation through the COSHAD/FrCOSHAD partial sum methods. To understand the convergence behaviour of the current methods whether independent of the parameters $p$ and $m$, the same calculation is conducted using other values. Here, the error, in the maximum norm, between the exact and the reconstructed solution as defined by

$$
L_{\infty}:=\max _{0<x_{i} \leq N}\left|f\left(x_{i}\right)-\tilde{f}_{m}\left(\bar{x}_{i}\right)\right|
$$

is used to demonstrate the numerical efficiency and convergence. Table 5 illustrates the logarithmic pointwise errors using present methods with respect to parameters $p$ and $m$. There is better convergence behaviour for all of the parameter selections.

The second example considers a more complex piecewise smooth signal $f_{2}\left(x_{i}\right)$ with length $N=256$ given by

$$
f_{2}\left(x_{i}\right)= \begin{cases}-1-\frac{\left(x_{i}-128\right)}{128}, & 0<x_{i} \leq 128, \\ \left(\frac{\left(x_{i}-129\right)}{128}-1\right)^{2}, & 128<x_{i} \leq 255,\end{cases}
$$

and there are two subintervals of equal length. The solid black line in Figure 9 shows the original piecewise function $f_{2}\left(x_{i}\right)$. Function $f_{2}\left(x_{i}\right)$ has the point of discontinuity at 128 . The partial sums of the COSHAD/FrCOSHAD series $f^{N_{d}}\left(x_{i}\right)$ with $N_{d}=5$ and $N_{d}=15$ can be found in Figure 9. The values of parameters $m$ and $p$ are chosen as $m=7$ for the COSHAD and $m=7$ and $p=0.6$ for the FrCOSHAD. Figure 9 shows that the approximations through the partial sum are inaccurate regardless of the COSHAD or the FrCOSHAD. However, the solution obtained by the proposed methods gives excellent results even for the small $N_{d}=3$ and $N_{d}=4$, which corresponds to their respective interval. In this experiment, we let the reconstruction coefficient vectors $\widetilde{\mathbf{g}}^{L}$ and $\widetilde{\mathbf{g}}^{R}$ denote $\widetilde{\mathbf{g}}$ in the left interval $[1,128]$ and in the right interval $[129,255]$, respectively. Exact reconstruction coefficient vectors can be obtained in terms of (51). The results are listed in Table 6, and the COSHAD and the FrCOSHAD coefficient matrix $\widehat{\mathbf{f}}^{L}$ and $\widehat{\mathbf{f}}^{R}$ computed using (47) is illustrated in Table 7.

The study found that the FrCOSHAD-based IPRM is not very sensitive to the fractional order $p$. Rather arbitrarily choosing $p=0.3$ and $p=0.5$ for the left and the right interval, the FrCOSHAD-based IPRM can also achieve accurate reconstructions of the discontinuous function $f_{2}\left(x_{i}\right)$ from their own spectra with $N_{d}=3$ and $N_{d}=4$ 


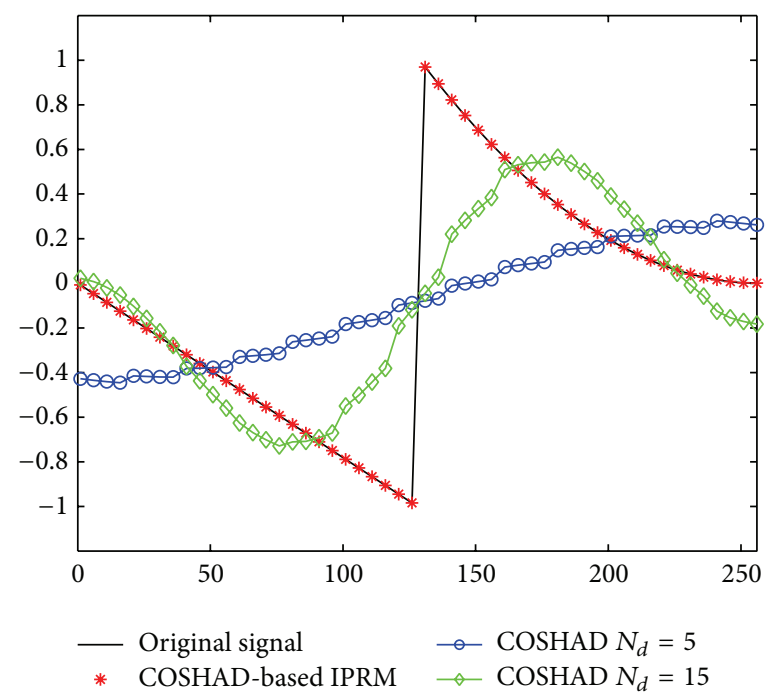

(a)

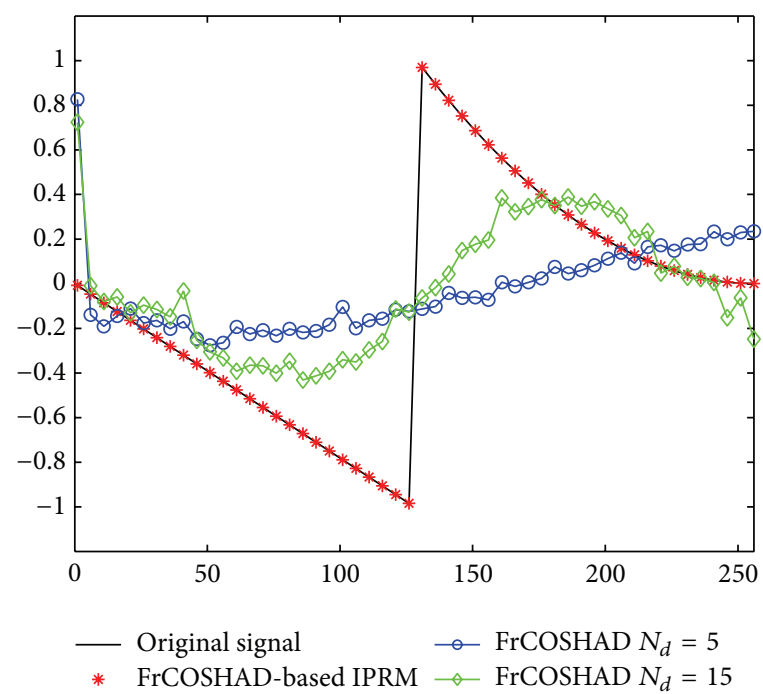

(b)

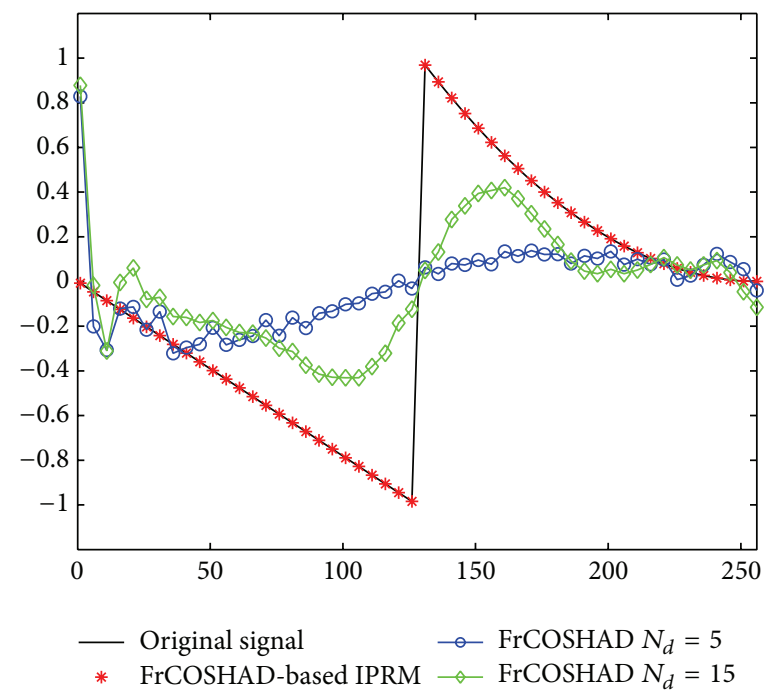

(c)

FIGURE 9: The function $f_{2}\left(x_{i}\right)$ and its approximation using the IPRM; (a) COSHAD-based IPRM, left interval: $u=2, m=6$; right interval: $u=3, m=2$; (b) FrCOSHAD-based IPRM, left interval: $u=2, m=6, p=0.6$, right interval: $u=3, m=2, p=0.4$; (c) FrCOSHAD-based IPRM, left interval: $u=2, m=6, p=0.3$, right interval: $u=3, m=2, p=0.5$.

TABLE 6: Reconstruction coefficient vectors $\widetilde{\mathbf{g}}$ for function $f_{2}\left(x_{i}\right)$.

\begin{tabular}{lcc}
\hline Method & $\widetilde{\mathbf{g}}^{L}\left(N_{d}=3, u=2, m=6, p=0.6\right)$ & $\widetilde{\mathbf{g}}^{R}\left(N_{d}=4, u=3, m=2, p=0.4\right)$ \\
\hline COSHAD-based IPRM & {$\left[\begin{array}{l}-0.50387500 \\
-0.49609375 \\
-0.00396751\end{array}\right]$} \\
\hline FrCOSHAD-based IPRM & {$\left[\begin{array}{c}-0.50390625-3.01387591 e-16 i \\
-0.335957845052 \\
-0.499969482421 \\
0.164072672526 \\
-3.5527136788 e-15\end{array}\right]$} \\
& {$\left[\begin{array}{c}-0.49609375-1.52252045 e-16 i \\
0.33595784-2.90958949 e-14 i \\
-0.49996948+2.38562478 e-14 i \\
0.16407267+4.39216256 e-14 i \\
9.76996261 e-15-2.85417475 e-14 i\end{array}\right]$} \\
\hline
\end{tabular}


TABLE 7: The COSHAD/FrCOSHAD coefficient matrix $\widehat{\mathbf{f}}$ for function $f_{2}\left(x_{i}\right)$.

\begin{tabular}{lcr}
\hline Method & $\widehat{\mathbf{f}}^{L}\left(N_{d}=3, u=2, m=6, p=0.6\right)$ & $\widehat{\mathbf{f}}^{R}\left(N_{d}=4, u=3, m=2, p=0.4\right)$ \\
\hline COSHAD-based IPRM & {$\left[\begin{array}{c}-8.06250000 \\
0.06250000 \\
4.58481289\end{array}\right]$} & {$\left[\begin{array}{l}21.583984375 \\
4.0312500000 \\
0.2500000000 \\
2.0156250000\end{array}\right]$} \\
FrCOSHAD-based IPRM & {$\left[\begin{array}{c}-5.93917075-1.18718599 e-15 i \\
0.22410421+4.03909759 e-16 i \\
0.97092404-7.30156437 e-16 i\end{array}\right]$} \\
\hline
\end{tabular}

TABLE 8: Reconstruction coefficient vectors $\widetilde{\mathbf{g}}$ for function $f_{3}\left(x_{i}\right)$.

\begin{tabular}{lcc}
\hline Method & $\widetilde{\mathbf{g}}^{L}\left(N_{d}=3, u=2, m=6, p=0.6\right)$ & $\tilde{\mathbf{g}}^{R}\left(N_{d}=4, u=3, m=2, p=0.4\right)$ \\
\hline COSHAD-based IPRM & {$\left[\begin{array}{l}-1.00775001 \\
-0.99218750 \\
-0.00793503\end{array}\right]$} & {$\left[\begin{array}{c}0.66404215 \\
0.49996948 \\
-0.16407267 \\
3.55271367 e-15\end{array}\right]$} \\
\hline FrCOSHAD-based IPRM & {$\left[\begin{array}{c}-1.00781250-6.02775183 e-16 i \\
-0.99218750-3.04509029 e-16 i \\
2.87547763 e-14+2.03306436 e-15 i\end{array}\right]$} \\
\hline
\end{tabular}

(see Figure $9(\mathrm{c})$ ). In contrast, the COSHAD/FrCOSHAD partial sum $f^{N_{d}}\left(x_{i}\right)$ with $N_{d}=5$ and $N_{d}=15$ gives poor approximations throughout the domain. The parameters used for the FrCOSHAD are $p=0.5$ and $m=7$, in this case.

In the last example, we consider another function $f_{3}\left(x_{i}\right)$ of the same length, which is piecewise smooth, defined by

$$
f_{3}\left(x_{i}\right)= \begin{cases}-1-\frac{\left(2 x_{i}-128\right)}{128}, & 0<x_{i} \leq 128 \\ 1-\left(\frac{\left(x_{i}-129\right)}{128}-1\right)^{2}, & 128<x_{i} \leq 255 .\end{cases}
$$

This function too has a jump discontinuity at value 128 . Plots of this function and the COSHAD/FrCOSHAD series approximation to $f_{3}\left(x_{i}\right)$ are shown in Figure 10. Again, this figure shows that the finite COSHAD/FrCOSHAD series expansion $f^{N_{d}}\left(x_{i}\right)$ is not good enough for $N_{d}=5$ and $N_{d}=15$. However, if the COSHAD/FrCOSHAD-based IPRM reconstructions are conducted by setting the same parameters used by the above example for the function $f_{3}\left(x_{i}\right)$, the exact reconstruction can be obtained, as expected. Similarly, the reconstruction coefficient vectors $\widetilde{\mathbf{g}}$ and series coefficient matrix $\widehat{\mathbf{f}}$ are calculated and listed in Tables 8 and 9 , respectively. These experimental results again demonstrate how the proposed approaches actually represent the piecewise smooth function $f_{3}\left(x_{i}\right)$.

We now return to the issue of the error analysis in the reconstruction process. Figure 11 illustrates the errors in the maximum norm, with a logarithmic scale. As might be expected, good convergence behaviour of the proposed methods can be observed because the maximum error $L_{\infty}$ is near zero. Furthermore, the convergence of this method is extremely rapid and requires only a few terms. However, beyond that, this figure again indicates that changing coefficients $p_{1}$ and $p_{2}$ has no obvious effect on the exponential convergence properties of the IPRM in the FrCOSHAD expansion.

\section{Conclusions}

In this paper, the discrete FrCOSHAD transform was defined and developed using the eigendecomposition method. By means of adjusting the parameter $m$, the developed FrCOSHAD transforms offer the advantages of both the DCT and the Hadamard by combining the optimal performance of the former with the ease of implementation of the latter. Several mathematical properties were discussed. Angle additivity and energy compaction capabilities were verified using the $1 \mathrm{D}$ and the $2 \mathrm{D}$ signals. The paper also proposed the quaternion COSHAD/FrCOSHAD transforms for colour images using the quaternion algebra. The main advantage of the quaternion strategy is that it provides the possibility of processing colour images by considering the three components of the colour RGB image as a whole, not separately. Block-based image reconstruction results demonstrated that the proposed transforms outperform some classical methods in qualitative and quantitative measurements.

Besides, a mathematical strategy for the 1D IPRM in the COSHAD/FrCOSHAD expansion was developed for the representation of the piecewise smooth functions from the information contained in a finite set of the COSHAD or the FrCOSHAD spectra. In this paper, we restricted ourselves to use the COSHAD/FrCOSHAD series expansion 
TABLE 9: The COSHAD/FrCOSHAD coefficient matrix $\widehat{\mathbf{f}}$ for function $f_{3}\left(x_{i}\right)$.

\begin{tabular}{lcr}
\hline Method & $\widehat{\mathbf{f}}^{L}\left(N_{d}=3, u=2, m=6, p=0.6\right)$ & $\widehat{\mathbf{f}}^{R}\left(N_{d}=4, u=3, m=2, p=0.4\right)$ \\
\hline COSHAD-based IPRM & {$\left[\begin{array}{c}-16.12500000 \\
0.12500000 \\
9.16962578\end{array}\right]$} & {$\left[\begin{array}{c}42.41601562 \\
-4.0312500000 \\
-0.2500000000 \\
-2.0156250000\end{array}\right]$} \\
FrCOSHAD-based IPRM & {$\left[\begin{array}{c}-11.87834150-2.37437198 e-15 i \\
0.44820843+8.07819519 e-16 i \\
1.94184809-1.46031287 e-15 i\end{array}\right]$}
\end{tabular}

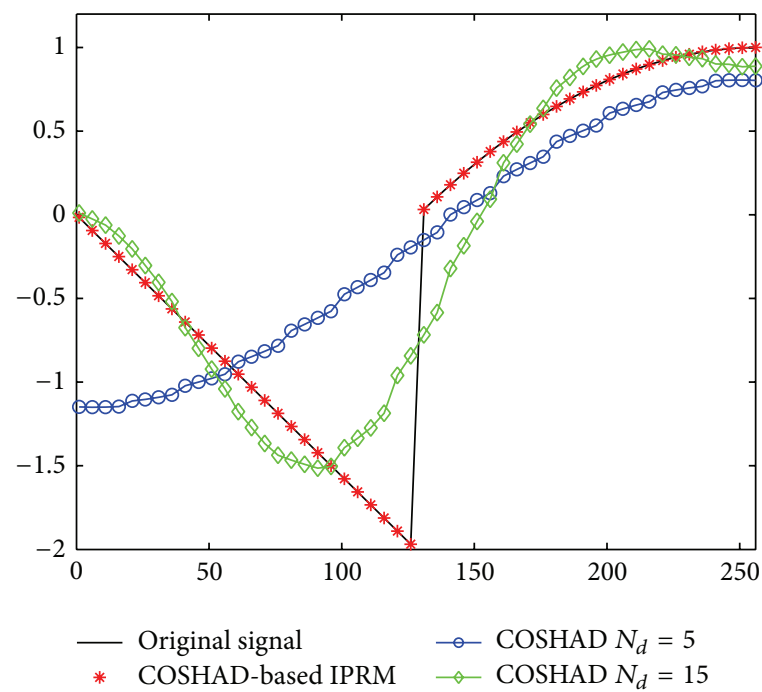

(a)

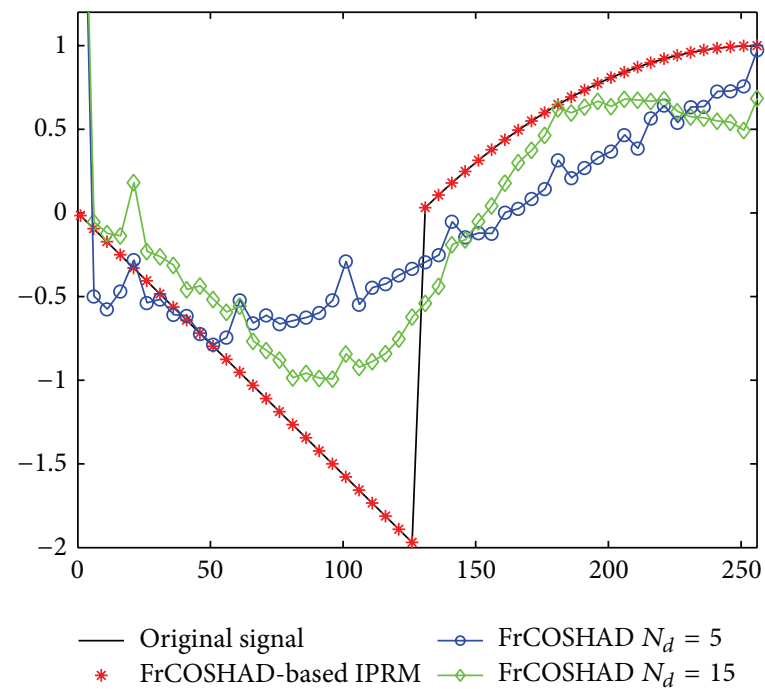

(b)

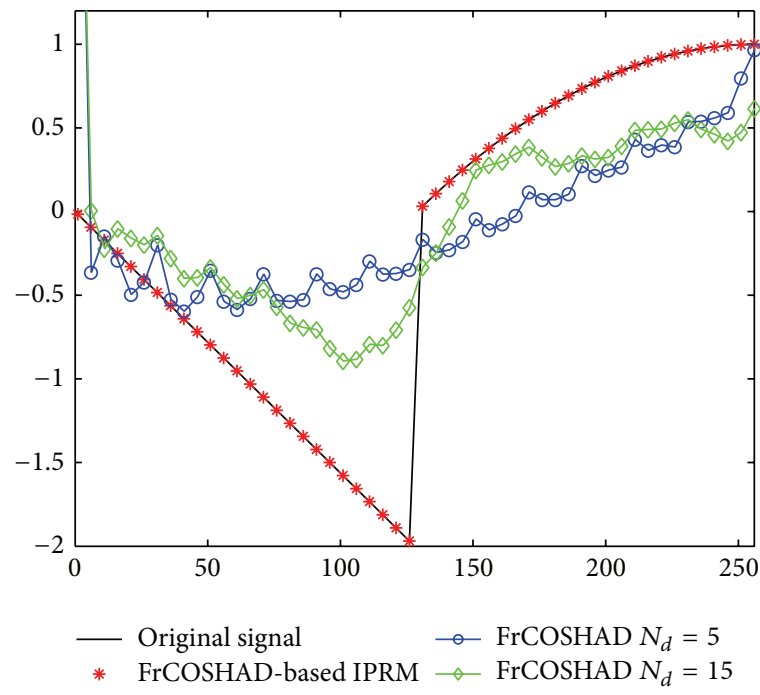

(c)

FIGURE 10: The function $f_{3}\left(x_{i}\right)$ and its approximation using the IPRM, (a) COSHAD-based IPRM, left interval: $u=2, m=6$; right interval: $u=3, m=2$; (b) FrCOSHAD-based IPRM, left interval: $u=2, m=6, p=0.6$, right interval: $u=3, m=2, p=0.4$; (c) FrCOSHAD-based IPRM, left interval: $u=2, m=6, p=0.3$, right interval: $u=3, m=2, p=0.5$. 


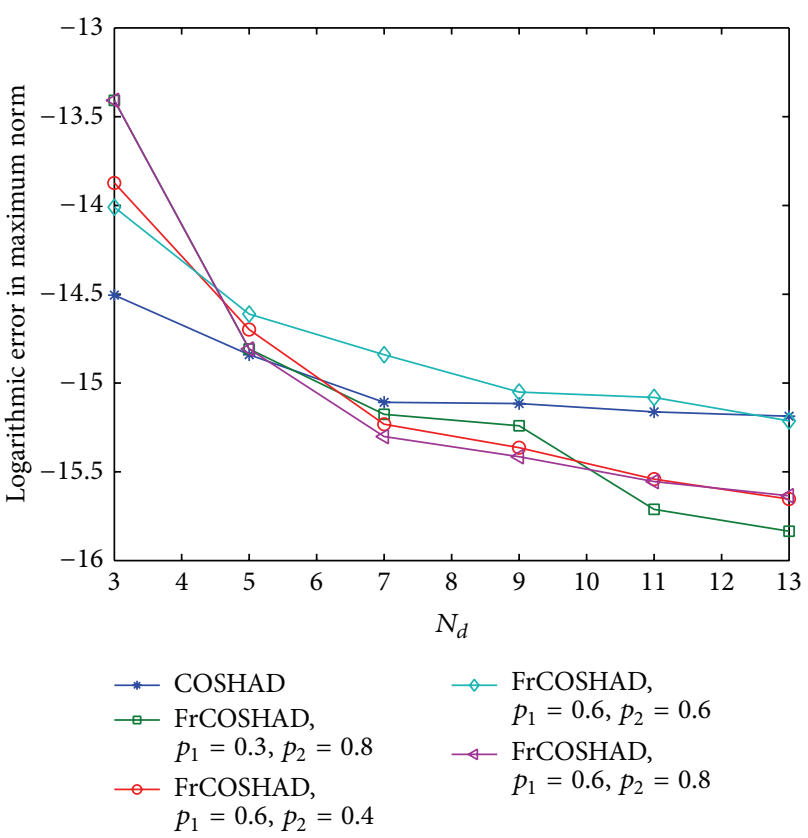

(a)

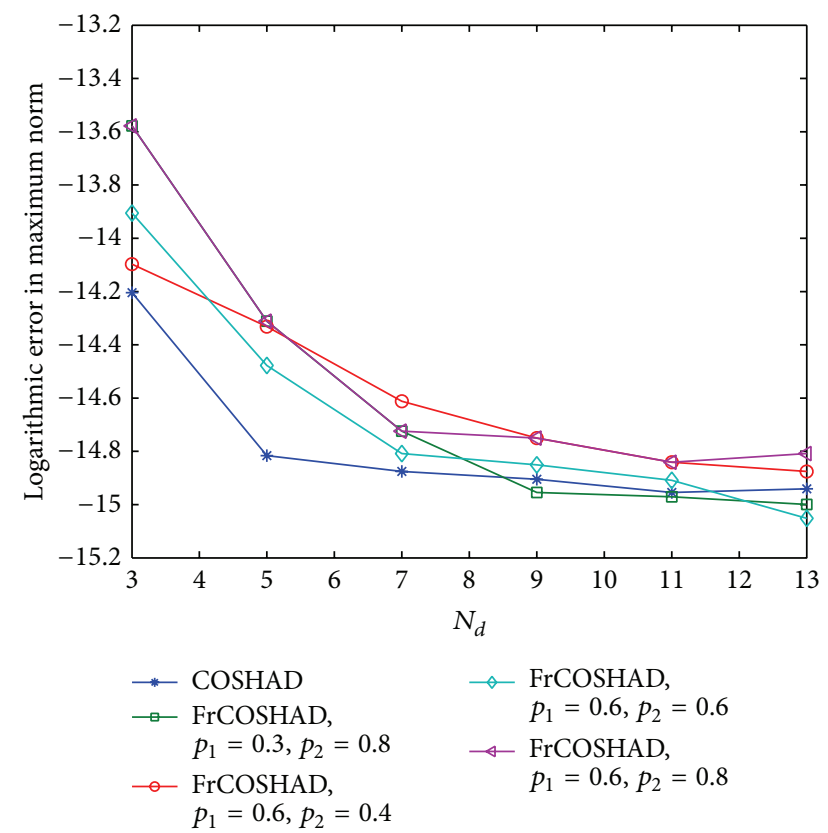

(b)

FIgURE 11: The errors in the maximum norm $L_{\infty}$ with different $p_{1}$ and $p_{2}$, in a logarithmic scale; left interval: $u=2, m=6$; right interval: $u=3, m=2$; (a) for function $f_{2}\left(x_{i}\right)$; (b) for function $f_{3}\left(x_{i}\right)$.

for description of the $1 \mathrm{D}$ signals because it is not difficult to extend to higher dimensions. Moreover, the paper provided a theoretical proof that the 1D COSHAD-based IPRM not only is spectrally convergent but also has independent parameters of its own.

Further investigations may include studying why, as fractional order increases, the trend of the PSNR values is almost a straight line under Gaussian white noise conditions (see
Figure 7). Is it coincidence, or is there a fundamental principle involved? Another question that should be addressed in future work is how to obtain other fractional orthogonal transforms using the Kronecker product methods.

\section{Conflict of Interests}

The authors declare that there is no conflict of interests regarding the publication of this paper.

\section{Acknowledgments}

The authors would like to thank the anonymous referees for their helpful comments and suggestions. This work was supported by the National Natural Science Foundation of China under Grant nos. 61371150, 61271357, and 61370174.

\section{References}

[1] F. M. Bayer and R. J. Cintra, "DCT-like transform for image compression requires 14 additions only," Electronics Letters, vol. 48, no. 15, pp. 919-921, 2012.

[2] R. J. Cintra and F. M. Bayer, "A DCT approximation for image compression," IEEE Signal Processing Letters, vol. 18, no. 10, pp. 579-582, 2011.

[3] S.-T. Wei, C.-W. Tien, B.-D. Liu, and J.-F. Yang, "Adaptive truncation algorithm for hadamard-transformed H.264/AVC lossless video coding," IEEE Transactions on Circuits and Systems for Video Technology, vol. 21, no. 5, pp. 538-549, 2011.

[4] A. Aung, B. P. Ng, and S. Rahardja, "Sequency-ordered complex Hadamard transform: properties, computational complexity and applications," IEEE Transactions on Signal Processing, vol. 56, no. 8, pp. 3562-3571, 2008.

[5] J. Wu, H. Shu, L. Wang, and L. Senhadji, "Fast algorithms for the computation of sliding sequency-ordered complex Hadamard transform," IEEE Transactions on Signal Processing, vol. 58, no. 11, pp. 5901-5909, 2010.

[6] G. Zeng and M. H. Lee, "A generalized reverse block jacket transform," IEEE Transactions on Circuits and Systems I: Regular Papers, vol. 55, no. 6, pp. 1589-1600, 2008.

[7] W. Ouyang and W.-K. Cham, "Fast algorithm for walsh hadamard transform on sliding windows," IEEE Transactions on Pattern Analysis \& Machine Intelligence, vol. 32, no. 8, pp. 165171, 2010.

[8] S. Pei, C. Tseng, M. Yeh, and J. Shyu, "Discrete fractional hartley and fourier transforms," IEEE Transactions on Circuits and Systems II: Analog and Digital Signal Processing, vol. 45, no. 6, pp. 665-675, 1998.

[9] S.-C. Pei and M.-H. Yeh, "The discrete fractional cosine and sine transforms," IEEE Transactions on Signal Processing, vol. 49, no. 6, pp. 1198-1207, 2001.

[10] S.-C. Pei and M.-H. Yeh, "Discrete fractional Hadamard transform," in Proceedings of the IEEE International Symposium on Circuits and Systems (ISCAS '99), vol. 3, pp. 179-182, June 1999.

[11] Y.-J. Yang, D. Baleanu, and X.-J. Yang, "Analysis of fractal wave equations by local fractional Fourier series method," Advances in Mathematical Physics, vol. 2013, Article ID 632309, 6 pages, 2013.

[12] C. C. Tseng, "Eigenvector and fractionalization of discrete hadamard transform," in Proceedings of the IEEE International 
Symposium on Circuits and Systems (ISCAS '07), pp. 2307-2310, May 2007.

[13] S. N. Merchant and B. V. Rao, "Signal processing via COSHAD transform," Computers and Electrical Engineering, vol. 12, no. 12, pp. 3-12, 1986.

[14] J. Jung and B. D. Shizgal, "On the numerical convergence with the inverse polynomial reconstruction method for the resolution of the Gibbs phenomenon," Journal of Computational Physics, vol. 224, no. 2, pp. 477-488, 2007.

[15] A. Abdi and S. M. Hosseini, "An investigation of resolution of 2-variate Gibbs phenomenon," Applied Mathematics and Computation, vol. 203, no. 2, pp. 714-732, 2008.

[16] J. Jung and B. D. Shizgal, "Inverse polynomial reconstruction of two dimensional Fourier images," Journal of Scientific Computing, vol. 25, no. 3, pp. 367-399, 2005.

[17] A. J. Laub, Matrix Analysis for Scientists \& Engineers, Society for Industrial and Applied Mathematics (SIAM), Philadelphia, Pa, USA, 2005.

[18] http://sipi.usc.edu/database/.

[19] T. A. Ell and S. J. Sangwine, "Hypercomplex Fourier transforms of color images," IEEE Transactions on Image Processing, vol. 16, no. 1, pp. 22-35, 2007.

[20] M. Vetterli, J. Kovačević, and V. Goyal, Foundations of Signal Processing, Cambridge University Press, 2013.

[21] H. Dadkhahi, A. Gotchev, and K. Egiazarian, "Inverse polynomial reconstruction method in DCT domain," EURASIP Journal on Advances in Signal Processing, vol. 2012, article 133, 2012.

[22] T. H. Fay and P. H. Kloppers, "The Gibbs phenomenon for series of orthogonal polynomials," International Journal of Mathematical Education in Science and Technology, vol. 37, no. 8, pp. 973-1000, 2006.

[23] B. Beckermann, V. Kalyagin, A. C. Matos, and F. Wielonsky, "How well does the Hermite-Padé approximation smooth the Gibbs phenomenon?" Mathematics of Computation, vol. 80, pp. 931-958, 2011.

[24] B. Beckermann, A. C. Matos, and F. Wielonsky, "Reduction of the Gibbs phenomenon for smooth functions with jumps by the $\varepsilon$-algorithm," Journal of Computational and Applied Mathematics, vol. 219, no. 2, pp. 329-349, 2008.

[25] D. Gottlieb and C.-W. Shu, "On the Gibbs phenomenon and its resolution,” SIAM Review, vol. 39, no. 4, pp. 644-668, 1997.

[26] B. D. Shizgal and J. Jung, "Towards the resolution of the Gibbs phenomena," Journal of Computational and Applied Mathematics, vol. 161, no. 1, pp. 41-65, 2003.

[27] J. Jung and B. D. Shizgal, "Generalization of the inverse polynomial reconstruction method in the resolution of the Gibbs phenomenon," Journal of Computational and Applied Mathematics, vol. 172, no. 1, pp. 131-151, 2004.

[28] H. S. Malvar, "Lapped transforms for efficient transform/subband coding," IEEE Transactions on Acoustics, Speech, and Signal Processing, vol. 38, no. 6, pp. 969-978, 1990.

[29] W. Feng and B. Hu, "Quaternion discrete Cosine transform and its application in color template matching," in Proceedings of the 1st International Congress on Image and Signal Processing (CISP '08), pp. 252-256, Sanya, China, May 2008.

[30] Z. Wang and A. C. Bovik, "A universal image quality index," IEEE Signal Processing Letters, vol. 9, no. 3, pp. 81-84, 2002. 


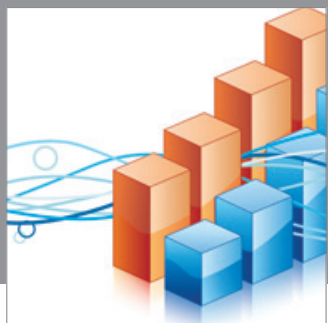

Advances in

Operations Research

mansans

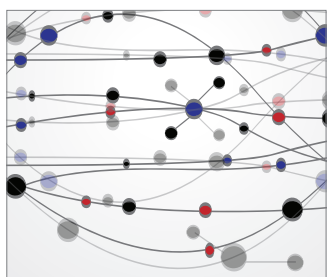

The Scientific World Journal
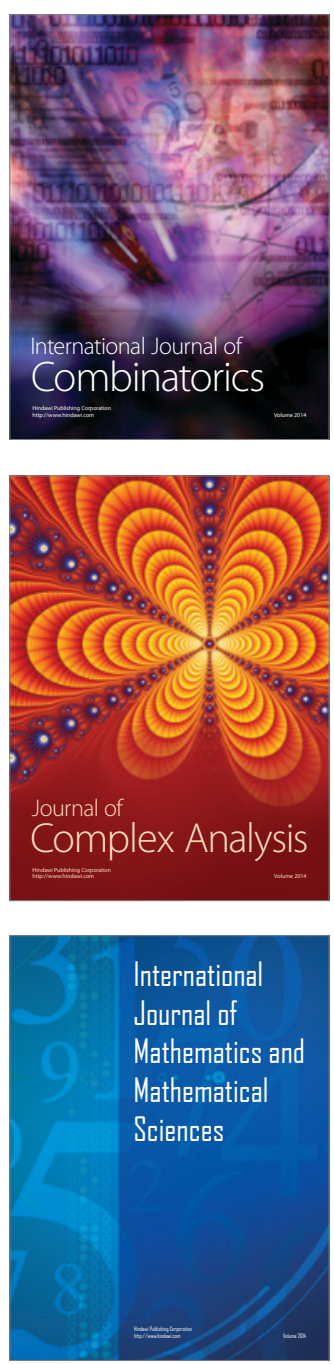
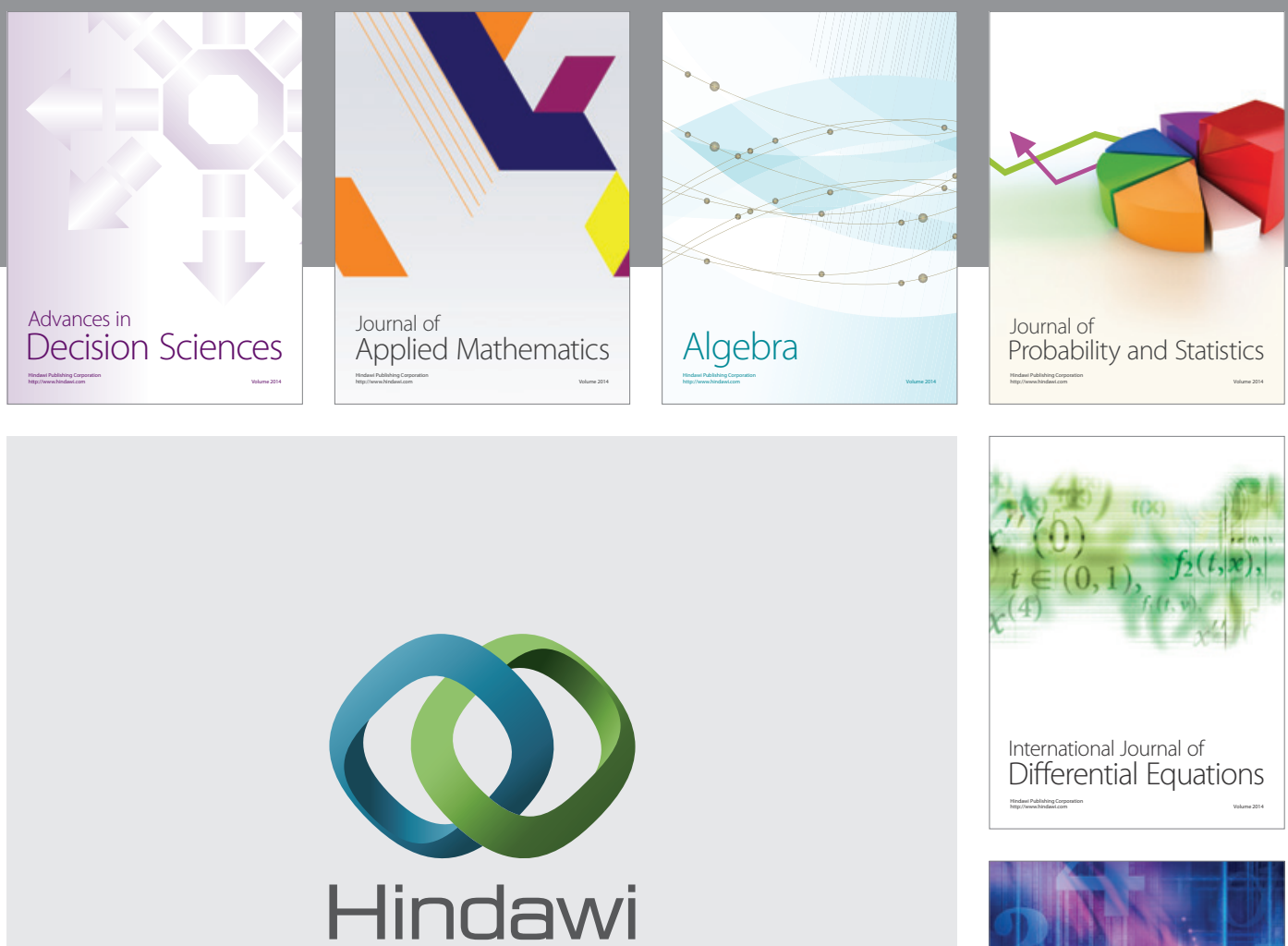

Submit your manuscripts at http://www.hindawi.com
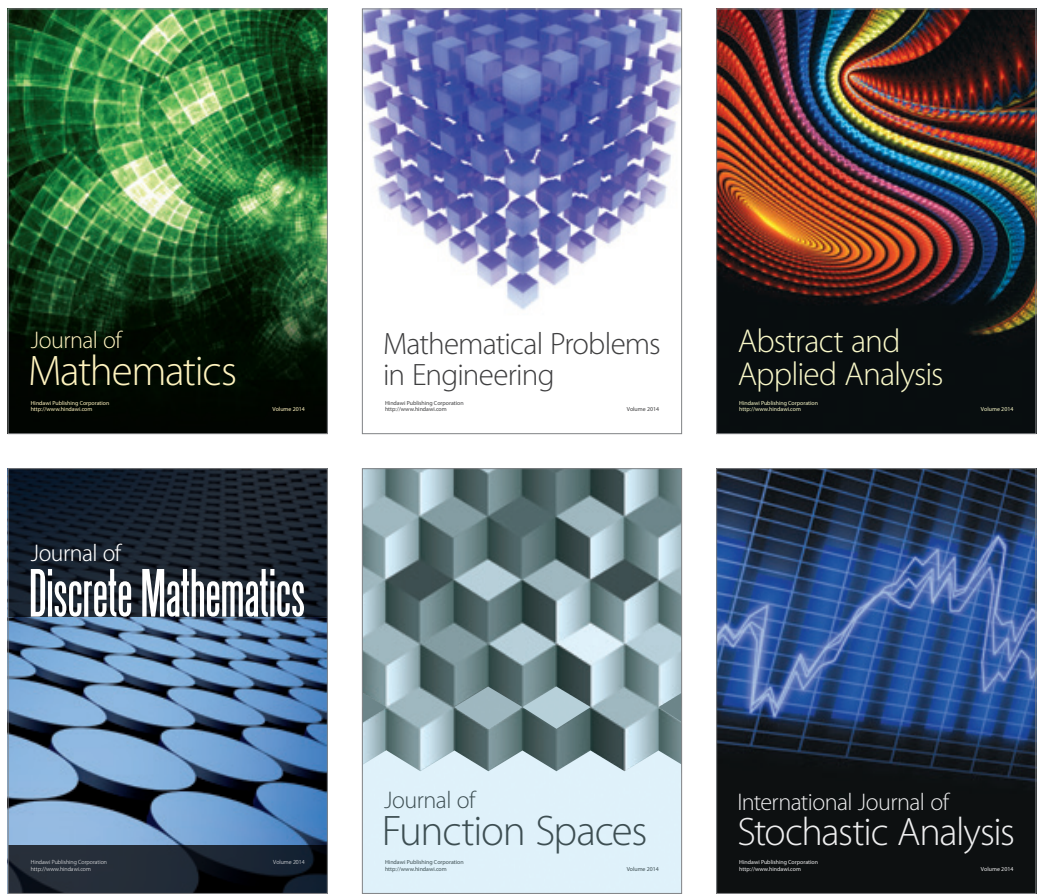

Journal of

Function Spaces

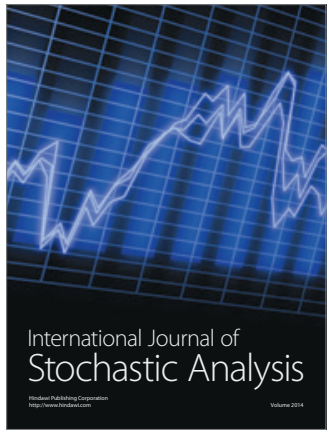

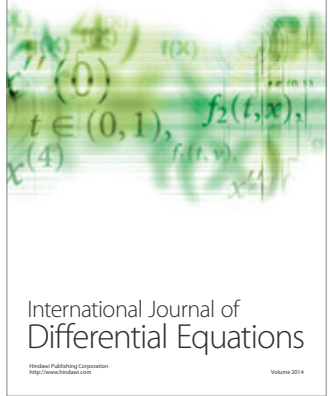
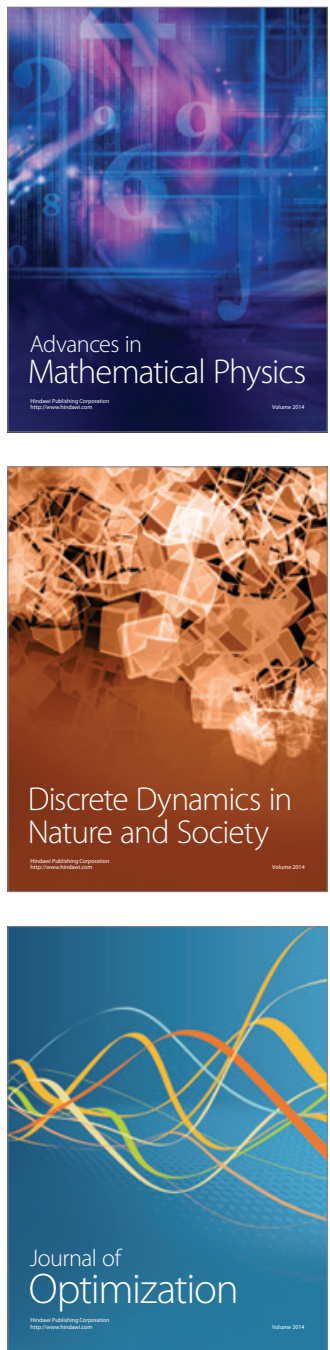